\title{
Band gaps of liquid water and hexagonal ice through advanced electronic-structure calculations
}

\author{
Thomas Bischoff $\odot,{ }^{*}$ Igor Reshetnyak $\odot$, and Alfredo Pasquarello $\odot$ \\ Chaire de Simulation à l'Echelle Atomique (CSEA), Ecole Polytechnique Fédérale de Lausanne (EPFL), 1015 Lausanne, Switzerland
}

(Received 2 December 2020; accepted 11 May 2021; published 4 June 2021)

\begin{abstract}
The fundamental band gaps of liquid water and hexagonal ice are calculated through advanced electronicstructure methods. We compare specifically the performance of state-of-the-art $G W$ calculations with nonempirical hybrid functionals. For the latter, we fix the free parameters either through the dielectric response of the material or through enforcing Koopmans' condition to localized states. The various approaches yield consistent band gaps, in good agreement with available experimental references. Furthermore, we discuss the critical aspects of each approach that underlie the band-gap predictions.
\end{abstract}

DOI: 10.1103/PhysRevResearch.3.023182

\section{INTRODUCTION}

Liquid water is a ubiquitous material in nature and has inevitably drawn great scientific attention. In particular, the electronic structure of liquid water has been the subject of numerous experimental [1-6] and theoretical studies [7-10]. These fundamental efforts enable a deeper understanding of liquid water in technological applications such as solvation processes [11-15] or catalytic reactions at solid-water interfaces [16-21]. In addition to the liquid phase, also various solid phases of water have been investigated. The precise knowledge of the electronic structure of these polymorphs is relevant for atmospheric science [22-26], geoscience [27], astronomy [28,29], and solid-state physics [30-33].

It is surprising that, despite this great scientific effort, the fundamental band gap of water is only known with mediocre accuracy. Indeed, the often-cited study of Bernas et al. reports a band gap for liquid water of $8.7 \pm 0.5 \mathrm{eV}$ [5]. This value and its sizable uncertainty reflect the lack of consensus among the various studies from which this band-gap estimate has been inferred. It is noteworthy that also the band gaps of the solid phases of water have not been determined with a high precision. In particular, the fundamental band gap of the most prominent ice Ih has been estimated to be $8.8 \pm 0.4 \mathrm{eV}$ [34]. Such substantial uncertainties call for further investigation.

Complementary to experimental studies, also a great deal of theoretical works have been devoted to this open question. Many-body perturbation theory in the $G W$ approximation is generally considered as the most accurate computational scheme for band-gap evaluation [35-38]. Various flavors of this approach have been instrumental in predicting the band gap of liquid water [7-9,16,39-41] and ice [42-44]. However,

\footnotetext{
*thomas.bischoff@epfl.ch

Published by the American Physical Society under the terms of the Creative Commons Attribution 4.0 International license. Further distribution of this work must maintain attribution to the author(s) and the published article's title, journal citation, and DOI.
}

the various studies have not reached a consensus on the band gap yet. Indeed, the reported values for liquid water range from 7.8 to $10.5 \mathrm{eV}$ with an uncertainty even larger than that of the experimental measurements. Among these studies, Chen et al. have deployed the most advanced method consisting in quasiparticle self-consistent $G W$ including vertex corrections and obtained a band gap of $8.9 \mathrm{eV}$ [8]. However, such high-level $G W$ calculations are computationally demanding and have remained rare. For further confirmation, it is therefore necessary to take into consideration a larger variety of electronic-structure approaches. Moreover, to allow for applications involving larger systems, it is highly desirable that such alternative methods are computationally less demanding.

Electronic-structure calculations based on hybrid functionals [45-48] can serve as a valuable alternative to $G W$ calculations and enable an accurate description of liquid water [10,11,14,49-52] and ice [49-52]. Despite these promising aspects, hybrid functionals have the drawback of containing undetermined parameters, such as the amount of incorporated Fock exchange. The empirical adjustment of these parameters remains unsatisfactory and is furthermore relying on accurate experimental references. To overcome these deficiencies, significant scientific effort has been devoted to the development of nonempirical hybrid-functional schemes. The basic idea consists in fixing the parameters of the adopted hybrid-functional forms by imposing properties of the exact functional. Two properties have been instrumental in achieving this target: (i) the asymptotically correct KohnSham potential [53], which reflects the long-range screening [54-56], and (ii) the piecewise linearity of the functional upon fractional electron occupation [57,58], also known as Koopmans' condition. The enforcement of these constraints has given rise to two main directions of research, which are identified either as dielectric-dependent hybrid (DDH) functionals [19,54,55,59-71] or as hybrid functionals satisfying Koopmans' condition [72-80]. Both approaches exhibit great potential due to the demonstrated accuracy achieved at moderate computational cost. In particular, DDH functionals have already been successfully applied to liquid 
water $[19,63,66,70]$. Similar investigations based on hybrid functionals satisfying Koopmans' condition are at present still lacking. A comparison of both nonempirical hybridfunctional schemes together with high-level $G W$ approaches might yield valuable insight into the fundamental band gap of water and ice.

In this work, we set out to study the fundamental band gap of liquid water and hexagonal ice through several advanced electronic-structure methods. These comprise state-of-the-art $G W$ schemes as well as nonempirical hybrid functionals. In particular, we consider quasiparticle self-consistent $G W$ (QSGW) calculations that account for vertex corrections in the screening and hybrid functionals in which the free parameters are fixed either through the dielectric response of the material or through enforcing Koopmans' condition to localized states. Both nonempirical procedures are applied to global and range-separated hybrid functionals. The evaluation of such a variety of different approaches within a consistent computational setup allows us to achieve a robust band-gap estimate and to overcome the spread of previous results in the literature. The comparison with available experimental references confirms the validity of our results.

This work is organized as follows. We review experimental data and establish robust references for our band-gap calculations in Sec. II. In Sec. III we describe the theoretical schemes used in this work. Section IV is devoted to computational aspects of our calculations. This comprises information about the structural models in Sec. IV A and computational details in Sec. IV B. In Sec. V we present the band gaps as obtained with the various advanced electronic-structure methods considered in this work. We summarize in Sec. VI.

\section{EXPERIMENTAL BAND-GAP REFERENCES}

Prior to deploying advanced electronic-structure calculations, it is our interest to establish robust experimental band-gap references. We infer such references through a critical review of various experimental studies in the literature. The fundamental band gap, defined as the separation between the valence-band maximum (VBM) and the conduction-band minimum $(\mathrm{CBM})$, is generally determined through the separate investigation of the two band edges. The VBM is given through the ionization potential (IP) as obtained in photoemission experiments. The CBM is determined by the electron affinity or equivalently by the position of the conduction band with respect to the vacuum level (denoted by $V_{0}$ ). These properties are generally accessed through inverse photoemission experiments or the analysis of thermodynamic data pertaining to the hydrated electron. We note that the fundamental band gap could also be inferred from the corresponding optical one [81-83]. However, this route requires a proper assessment of the excitonic binding energy for which a final consensus has not yet been reached $[39,42,83,84]$. Therefore, we do not include optical spectroscopy experiments in our analysis of band-gap references.

First, we examine the fundamental band gap of liquid water at ambient temperature. Early studies by Delahay and coworkers reported a photoemission threshold of $9.3 \pm 0.3 \mathrm{eV}$ [3], which was later revised to $10.06 \mathrm{eV}$ [4]. The more recent work of Winter et al. indicated a very similar value of $9.9 \mathrm{eV}$
[6]. Based on the latest results, we assume $10.0 \pm 0.1 \mathrm{eV}$ for the ionization potential of liquid water. For the unoccupied states, Bernas et al. reported $V_{0}=-1.2 \mathrm{eV}$ [2], later revised to $-0.74 \mathrm{eV}$ [5]. A recent study by Ambrosio et al. inferred $V_{0}=-0.97 \mathrm{eV}$ from thermodynamical data for the hydrated electron [14]. Overall, we estimate $V_{0}=-1.0 \pm 0.2 \mathrm{eV}$. We do not consider the value of $V_{0}=-0.12 \mathrm{eV}$ proposed by Coe et al. [85] due to the criticism raised in Ref. [14]. Altogether, we deduce a fundamental band gap for liquid water of $9.0 \pm 0.2 \mathrm{eV}$. This value is consistent with previous works by Bernas et al. and Painter et al., which reported band gaps of $8.7 \pm 0.5 \mathrm{eV}$ [5] and $9 \mathrm{eV}$ [86], respectively. Moreover, the present estimation is based on more recent experimental studies and subject to a smaller uncertainty. We remark that a fundamental band gap of 9.1-9.2 eV was also inferred in Ref. [10].

Next we consider the fundamental band gap of ice. Among the various possible phases [87], we are particularly interested in hexagonal ice Ih. This phase is stable at ambient pressure and has been subject to numerous experimental and computational studies. To benchmark the fundamental band gap of Ih, we adopt in the following also results obtained for polycrystalline and amorphous ice structures. This is justified through the studies of Nordlund et al. [88] and Kobayashi [82], which have demonstrated a remarkable similarity in the electronic structure of crystalline and amorphous ice based on photoemission and optical spectroscopy, respectively. Furthermore, we note that the considered experimental results have generally been obtained at a temperature of $77 \mathrm{~K}$. The measurements in Refs. [88-90] were performed at a slightly higher temperature of 90-103 K.

Early photoemission experiments by Shibaguchi et al. indicated an ionization potential of $10.5 \mathrm{eV}$ [89]. This result has been confronted with the value of $8.7 \pm 0.1 \mathrm{eV}$ obtained by Baron et al. [91] and that of $8.8 \mathrm{eV}$ by Campbell et al. [92]. However, it should be considered that the latter studies define the onset of the photoemission spectra according to an extended tail at lower energies. Instead, the linearly extrapolated threshold reveals a value of $\sim 10 \mathrm{eV}$ [92], in much better agreement with that of Shibaguchi et al. [89]. Furthermore, from the work of Pache et al. [93], one also infers an IP of $\sim 10 \mathrm{eV}$ [94]. More recent photoemission studies of Winter et al. [6] and Nordlund et al. [88] support these values for the ionization potential. Specifically, Winter et al. remarked that electron binding energies (and thus the IP) in ice tend to be larger by $\sim 0.1-1.0 \mathrm{eV}$ than those obtained for liquid water $(10.0 \pm 0.1 \mathrm{eV})$. We account for these various experimental measurements by adopting an IP estimate of $10.3 \pm 0.3 \mathrm{eV}$. For the conduction-band edge, early studies of Baron et al. and Grand and Bernas reported rather distinct values of $V_{0}=-0.9 \mathrm{eV}$ [91] and $-0.1 \mathrm{eV}$ [95], respectively. Later, Michaud et al. derived a value of $V_{0}=-1 \mathrm{eV}$ [94] on the basis of the photoelectric threshold [96] and the photoconductivity [97] of hydrated electrons. Most recently, Stähler et al. measured $V_{0}=-0.8 \mathrm{eV}$ through time-resolved photoemission experiments [90]. Overall, we take on the value of $V_{0}=-0.9 \pm 0.1 \mathrm{eV}$, only marginally higher than the one assumed for liquid water. Based on the present discussion of IP and $V_{0}$, we infer a fundamental band gap for hexagonal ice of $9.4 \pm 0.3 \mathrm{eV}$. This estimate is in overall agreement 
TABLE I. Fundamental band gaps $E_{\mathrm{g}}^{\text {expt }}$ (in $\mathrm{eV}$ ) of liquid water $\ell-\mathrm{H}_{2} \mathrm{O}$ and hexagonal ice $\mathrm{Ih}$ as inferred from a review of various experimental data. The given band gaps correspond to temperatures of 300 and $77 \mathrm{~K}$, respectively. Band-gap corrections $E_{\mathrm{g}}^{\text {corr }}$ (in eV) are also given. These corrections account for the band-gap reduction due to nuclear quantum effects in liquid water and to the zero-phonon renormalization in hexagonal ice.

\begin{tabular}{lcc}
\hline \hline Phase of water & $E_{\mathrm{g}}^{\text {expt }}$ & $E_{\mathrm{g}}^{\text {corr }}$ \\
\hline$\ell-\mathrm{H}_{2} \mathrm{O}$ & $9.0 \pm 0.2$ & $0.7^{\mathrm{a}}$ \\
$\mathrm{Ih}$ & $9.4 \pm 0.3$ & $1.5^{\mathrm{b}}$ \\
\hline \hline
\end{tabular}

${ }^{\mathrm{a}}$ Reference [8], $T=300 \mathrm{~K}$.

${ }^{\mathrm{b}}$ References [34,98], $T=0 \mathrm{~K}$.

with earlier studies by Michaud et al. and Engel et al., which reported band gaps of $\sim 9 \mathrm{eV}$ [94] and $8.8 \pm 0.4 \mathrm{eV}$ [34], respectively. The differences result from a more stringent review of the recent literature in the present work.

Overall, we adopt a band-gap reference for liquid water at $300 \mathrm{~K}$ of $9.0 \pm 0.2 \mathrm{eV}$. For hexagonal ice at $77 \mathrm{~K}$, we estimate a band gap of $9.4 \pm 0.3 \mathrm{eV}$. We observe a rather small difference of $0.4 \mathrm{eV}$ on average between these two phases of water. This observation indicates that the fundamental band gap of water depends only weakly on temperature and crystal structure. A similar conclusion was reached by Bernas et al. [5]. In this work, the band gap of liquid water is calculated through configurations obtained at $300 \mathrm{~K}$. For hexagonal ice, we obtain the band gap through a calculation at relaxed atomic positions. The comparison of the latter with the experimental band-gap references at $77 \mathrm{~K}$ is justified insofar as the finite temperature difference is expected to have a negligible effect. Indeed, Engel et al. have shown that the calculated band gap of Ih is almost independent of temperature between 0 and $77 \mathrm{~K}$ [34]. Estimated variations are limited to $\sim 0.02-0.05 \mathrm{eV}$ and are therefore disregarded [34].

After the critical review of experimental band-gap references, we discuss further effects that have to be taken into account to ensure a consistent comparison between theory and experiment. In particular, we consider nuclear quantum effects (NQE) in liquid water and zero-phonon renormalization (ZPR) in hexagonal ice. These effects originate from the quantum mechanical nature of the nuclei and generally induce a renormalization of the band gap. Since we consider classical nuclei in our structural models (cf. Sec. IV A), these renormalizations are added as a posteriori corrections to the band gaps calculated with the advanced electronic-structure methods. A summary of the band-gap references and corrections adopted in this work is given in Table I.

For liquid water, various studies have shown that the NQE significantly affect the electronic structure $[8,9,41,99]$. Indeed, Chen et al. found a band-gap reduction of $0.7 \mathrm{eV}$ due to $\mathrm{NQE}$, consistently for various state-of-the-art $G W$ approaches [8]. For the semilocal Perdew-Burke-Ernzerhof (PBE) functional [100], this effect reduces to $0.5 \mathrm{eV}$ [8]. These results are in overall agreement with other studies in the literature. The NQE have been found to be $0.5 \mathrm{eV}$ with the $G_{0} W_{0} @ \mathrm{RSH}$ method [9], $0.6 \mathrm{eV}$ with the PBE0-ADMM-D3 hybrid functional [99], and $0.65 \mathrm{eV}$ with eigenvalue self-consistent $G W$ calculations (evGW) [41]. Among these various values, we adopt throughout this work the $G W$ value from Chen et al. for the band-gap correction due to NQE. This choice is motivated, on the one hand, by the fact that this estimate results from one of the most accurate theoretical schemes and, on the other hand, by the fact that we adopt in this work the very same structural configurations obtained by Chen et al. [101]. Therefore, we correct the band gap of liquid water at ambient temperature by $0.7 \mathrm{eV}$ due to NQE. To provide further justification for this way of proceeding, we additionally compute the NQE with the most accurate hybrid-functional schemes considered in this work. More specifically, we employ the functionals $\operatorname{CAM}\left(0,1 / \varepsilon_{\infty}^{\mathrm{sc}}, \mu_{\mathrm{TF}}\right)$ and $\operatorname{CAM}\left(\alpha_{\mathrm{s}, \mathrm{K}}, 1 / \varepsilon_{\infty}^{\text {expt }}, \mu_{\mathrm{TF}}\right)$, which are described later in Secs. VB and V C, respectively. These functionals yield bandgap reductions due to NQE of 0.63 and $0.73 \mathrm{eV}$, respectively. These corrections are in good accord with the result of Chen et al. [8]. This agreement further justifies the use of a band-gap correction of $0.7 \mathrm{eV}$ for the $\mathrm{NQE}$ in liquid water.

In analogy to liquid water, we also consider the ZPR of the band gap in the case of hexagonal ice. Monserrat et al. calculated a ZPR of $1.52 \mathrm{eV}$ by means of density functional theory (DFT) calculations at the semilocal and hybrid functional levels [98]. Engel et al. confirmed this estimate in an extended study involving various proton orderings in hexagonal ice [34]. Therefore, we adopt throughout this work the value of $1.52 \mathrm{eV}$ for the a posteriori correction of the calculated band gaps in hexagonal ice.

It is noteworthy that the band-gap corrections for the two considered water phases are significantly different. Indeed, the ZPR of the band gap of hexagonal ice is larger than the $\mathrm{NQE}$ on the band gap of liquid water by $\sim 0.8 \mathrm{eV}$. Engel et al. have found that the ZPR of hexagonal ice varies by only $\sim 0.1 \mathrm{eV}$ over the temperature range $0-240 \mathrm{~K}$ [34]. Therefore, the dominating part of this effect cannot solely be assigned to the temperature difference between the two phases, but should rather be related to the underlying atomic structures. In particular, in the case of liquid water, the band-gap reduction results from a complex interplay between the broadenings in the density of states due to the NQE and to the molecular disorder.

\section{THEORETICAL SCHEMES}

\section{A. Quasiparticle self-consistent $\boldsymbol{G W}$}

The many-body perturbation theory in Hedin's $G W$ approximation is one of the most elaborate schemes to describe the electronic structure of materials [35]. In recent decades, it has evolved into a commonly accepted standard for bandgap calculations. In the following, we briefly review various flavors of this technique that are currently in use.

Technically, $G W$ calculations are often carried out as a perturbative correction to wave functions and eigenvalues obtained from DFT [102,103]. This so-called one-shot $G W\left(G_{0} W_{0}\right)$ correction generally improves the band gaps $[104,105]$ with respect to the underestimated ones from semilocal DFT [106]. However, this approach remains unsatisfactory insofar as it strongly depends on the considered 


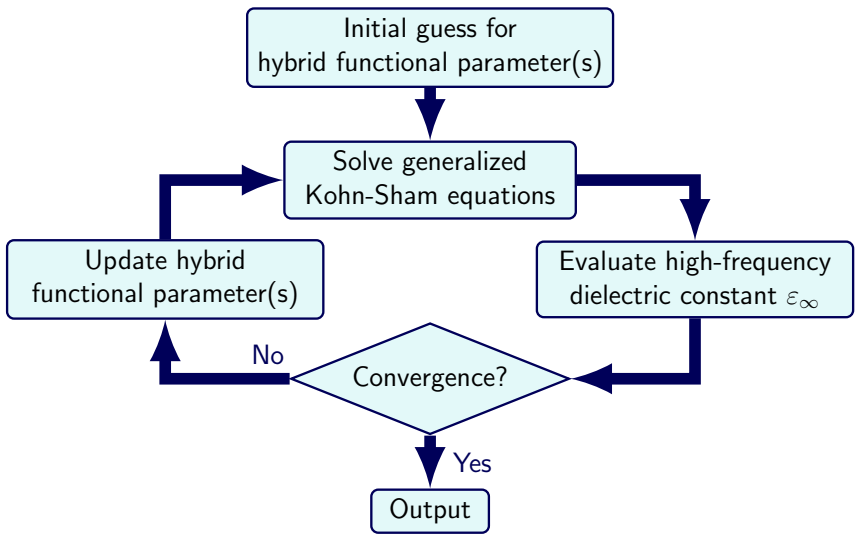

FIG. 1. Workflow for the construction of dielectric-dependent hybrid functionals.

starting point [104]. In case the initial wave functions give an inaccurate description, the $G_{0} W_{0}$ corrections might fail notably $[107,108]$. To overcome this deficiency, various $G W$ schemes have been developed in which perturbative corrections are iterated until a self-consistent description is reached [36,108-112].

One prominent representative of a self-consistent $G W$ scheme is the QSGW method [36,109,111]. It is based on a Hermitian ansatz for the $G W$ self-energy and has given moreaccurate band gaps and bandwidths than fully self-consistent $G W[36,113]$. However, it was observed that QSGW systematically overestimates the band gap as a consequence of the missing electron-hole interaction [37,38]. This obstacle has been circumvented through the incorporation of vertex corrections in the screened interaction $\tilde{W}[37,38,114]$ resulting in the QSGW scheme. The accuracy of this approach has been verified for numerous materials such as common semiconductors and insulators [37,38,115], transition-metal compounds [38,116,117], alkali-metal halides [75], metalhalide perovskites [80,118], and liquid water [8]. It is therefore generally acknowledged that QS $G \tilde{W}$ ranks among the most reliable $G W$ approaches for band-gap predictions.

\section{B. Dielectric-dependent hybrid functionals}

The second advanced electronic-structure method investigated in this work consists in dielectric-dependent hybrid functionals. In this approach, the dielectric response of the considered material is used to determine the free hybrid-functional parameters. In particular, the inverse highfrequency dielectric constant $1 / \varepsilon_{\infty}$ has been shown to be in close relation with the incorporated amount of Fock exchange [54,55]. The extension of this basic finding has led to various DDH schemes differing in technical aspects such as selfconsistency $[60,63,64]$, range separation $[66,68,69]$, or spatial variability [19].

In this study, we determine DDH functionals through the workflow visualized in Fig. 1. First, after an initial guess of the hybrid-functional parameters is made, the generalized Kohn-Sham (GKS) equations [119] are solved and the high-frequency dielectric constant $\varepsilon_{\infty}$ is evaluated. Then the inverse of this quantity is used to obtain an updated set of hybrid-functional parameters. These steps are iterated until a self-consistent description is reached. We note that the parameter update in Fig. 1 is specific to the adopted class of hybrid functionals. We discuss this aspect separately for the different classes of hybrid functionals investigated in this work.

First, we consider the global hybrid functional $\operatorname{PBE0}(\alpha)$, which incorporates at all interaction distances the same fraction $\alpha$ of Fock exchange [46]. The nonlocal exchange potential of this functional reads

$$
V_{x}\left(\mathbf{r}, \mathbf{r}^{\prime}\right)=\alpha V_{x}^{\mathrm{Fock}}\left(\mathbf{r}, \mathbf{r}^{\prime}\right)+(1-\alpha) V_{x}^{\mathrm{PBE}}(\mathbf{r}) \delta\left(\mathbf{r}-\mathbf{r}^{\prime}\right),
$$

where $V_{x}^{\text {Fock }}$ denotes the nonlocal Fock exchange potential and $V_{x}^{\mathrm{PBE}}$ represents the semilocal PBE exchange potential. The correlation in the $\operatorname{PBE} 0(\alpha)$ functional is included at the PBE level of theory. Employing the DDH scheme, the mixing parameter $\alpha$ is determined in the standard fashion through $\alpha=$ $1 / \varepsilon_{\infty}$. The self-consistent procedure in Fig. 1 then converges to a dielectric constant $\varepsilon_{\infty}^{\mathrm{sc}}$ and a mixing parameter $\alpha=1 / \varepsilon_{\infty}^{\mathrm{sc}}$. The hybrid functional determined in this way is denoted by $\operatorname{PBE0}\left(1 / \varepsilon_{\infty}^{\mathrm{sc}}\right)$. It is noteworthy that different notations for this functional have been used in the literature, such as sc-hybrid [63], DD-PBEH [69], and DD-PBE0 [80].

Second, we derive the functional $\operatorname{CAM}\left(\alpha_{\mathrm{s}}, \alpha_{\ell}, \mu\right)$ from the functional $\operatorname{PBE} 0(\alpha)$ following the Coulomb-attenuating method (CAM) of Yanai et al. [48]. This range-separated hybrid functional offers a substantial amount of flexibility through the appearance of three parameters. The rangeseparation parameter $\mu$ determines the partitioning of the Coulomb potential through the use of an error function:

$$
\frac{1}{\left|\mathbf{r}-\mathbf{r}^{\prime}\right|}=\frac{\operatorname{erfc}\left(\mu\left|\mathbf{r}-\mathbf{r}^{\prime}\right|\right)}{\left|\mathbf{r}-\mathbf{r}^{\prime}\right|}+\frac{\operatorname{erf}\left(\mu\left|\mathbf{r}-\mathbf{r}^{\prime}\right|\right)}{\left|\mathbf{r}-\mathbf{r}^{\prime}\right|}
$$

The first and the second term on the right-hand side of Eq. (2) determine the short-range and the long-range part of the exchange potential, respectively. The exchange potential is then determined starting from the semilocal PBE expression and admixing distinct fractions of nonlocal Fock exchange in the two regimes. We use the fraction $\alpha_{\mathrm{s}}\left(\alpha_{\ell}\right)$ for the admixture in the short (long) range. The resulting exchange potential of the functional $\operatorname{CAM}\left(\alpha_{\mathrm{s}}, \alpha_{\ell}, \mu\right)$ then reads

$$
\begin{aligned}
V_{x}\left(\mathbf{r}, \mathbf{r}^{\prime}\right)= & {\left[\alpha_{\mathrm{s}}+\left(\alpha_{\ell}-\alpha_{\mathrm{s}}\right) \operatorname{erf}\left(\mu\left|\mathbf{r}-\mathbf{r}^{\prime}\right|\right)\right] V_{x}^{\mathrm{Fock}}\left(\mathbf{r}, \mathbf{r}^{\prime}\right) } \\
& +\left\{1-\left[\alpha_{\mathrm{s}}+\left(\alpha_{\ell}-\alpha_{\mathrm{s}}\right) \operatorname{erf}\left(\mu\left|\mathbf{r}-\mathbf{r}^{\prime}\right|\right)\right]\right\} V_{x}^{\mathrm{PBE}}(\mathbf{r}) .
\end{aligned}
$$

It is noteworthy that this functional reverts to $\operatorname{PBE} 0(\alpha)$ for $\alpha=$ $\alpha_{\mathrm{s}}=\alpha_{\ell}$ irrespective of the $\mu$ parameter. Furthermore, one can distinguish two limiting regimes for the range separation. For $\mu \rightarrow 0$, one finds the $\operatorname{PBE} 0\left(\alpha_{\mathrm{s}}\right)$ functional. Analogously, for $\mu \rightarrow \infty$, the CAM functional converges asymptotically to $\operatorname{PBE} 0\left(\alpha_{\ell}\right)$. In addition, the functional form given in Eq. (3) also encompasses various commonly used hybrid functionals. For instance, the Heyd-Scuseria-Ernzerhof functional is obtained for $\alpha_{\mathrm{s}}=0.25, \alpha_{\ell}=0$, and $\mu=0.11 \mathrm{bohr}^{-1}[47,120]$.

The three parameters of the functional $\operatorname{CAM}\left(\alpha_{\mathrm{s}}, \alpha_{\ell}, \mu\right)$ are determined as follows within the self-consistent DDH procedure. The short-range fraction $\alpha_{\mathrm{s}}$ is taken as a constant for which different values have been proposed in the literature. Chen et al. applied $\alpha_{\mathrm{s}}=1$ with the underlying idea of 


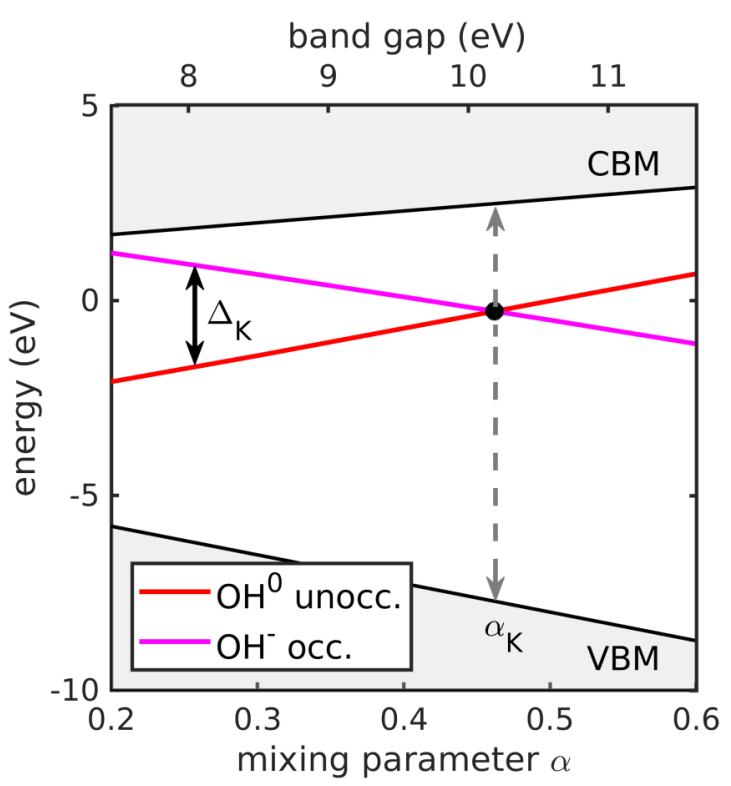

FIG. 2. Occupied and unoccupied single-particle energy levels of the $\mathrm{OH}$ radical in hexagonal ice as a function of the mixing parameter $\alpha$ used in the $\operatorname{PBE} 0(\alpha)$ functional. The deviation from Koopmans' condition $\Delta_{\mathrm{K}}$ corresponds to the separation between these energy levels and varies with $\alpha$. The point of intersection of the two singleparticle energy levels corresponds to the enforcing of Koopmans' condition and is denoted by $\alpha_{\mathrm{K}}$. The band edges vs $\alpha$ are also shown. The dashed vertical line indicates the resulting band-gap estimate prior to the application of the corrections discussed in Sec. II.

achieving a hybrid functional that reproduces the fully unscreened Coulomb interaction in the short range [69]. Skone et al. used instead an attenuated short-range exchange based on $\alpha_{\mathrm{s}}=0.25$ [66]. In this work, we consider both ways of fixing $\alpha_{\mathrm{s}}$ and compare the resulting implications for the construction of range-separated DDH functionals. Moreover, we investigate the effect of adopting purely semilocal exchange in the short range as obtained by setting $\alpha_{\mathrm{s}}=0$. This definition is motivated by the success of long-range corrected hybrid functionals [121-125] and will be discussed in more detail in Sec. VB. The second parameter of the CAM func- (a)

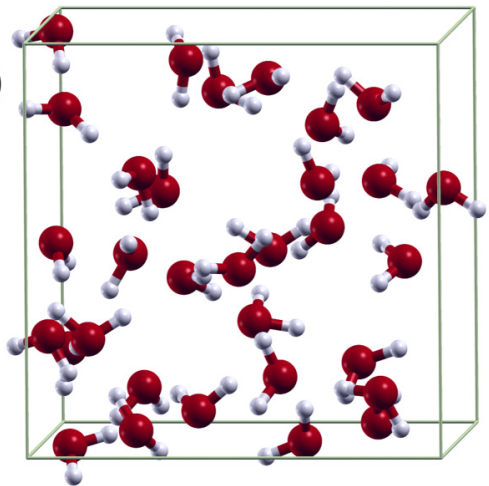

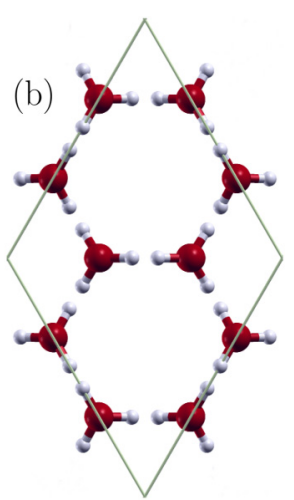

FIG. 3. Graphical illustration of (a) an instantaneous configuration of liquid water and (b) the Bernal-Fowler model of hexagonal ice as viewed along the $c$ axis. Oxygen and hydrogen atoms are shown in red and white, respectively.
TABLE II. Comparison of the range-separated DDH functionals considered in this work with respect to similar approaches reported in the literature: DD-RSH-CAM [69], DSH [68], and RS-DDH [66]. The comparison is made in terms of the fraction of short-range Fock exchange $\alpha_{\mathrm{s}}$, the fraction of long-range Fock exchange $\alpha_{\ell}$, and the range-separation parameter $\mu$.

\begin{tabular}{lccc}
\hline \hline Functional & $\alpha_{\mathrm{s}}$ & $\alpha_{\ell}$ & $\mu$ \\
\hline $\mathrm{CAM}\left(1,1 / \varepsilon_{\infty}^{\mathrm{sc}}, \mu_{\mathrm{TF}}\right)^{\mathrm{a}}$ & 1 & $1 / \varepsilon_{\infty}^{\mathrm{sc}}$ & $\mu_{\mathrm{TF}}$ \\
$\mathrm{DD}-\mathrm{RSH}-\mathrm{CAM}^{\mathrm{b}}$ & 1 & $1 / \varepsilon_{\infty}^{\mathrm{sc}}$ & $\mu_{\mathrm{fit}}^{\mathrm{scc}}$ \\
$\mathrm{DSH}^{\mathrm{d}}$ & 1 & $1 / \varepsilon_{\infty}^{\mathrm{sc}}$ & $\mu_{\mathrm{eff}}^{\mathrm{sc}}{ }^{\mathrm{e}}$ \\
$\mathrm{CAM}^{\mathrm{s}}\left(0.25,1 / \varepsilon_{\infty}^{\mathrm{sc}}, \mu_{\mathrm{TF}}\right)^{\mathrm{a}}$ & 0.25 & $1 / \varepsilon_{\infty}^{\mathrm{sc}}$ & $\mu_{\mathrm{TF}}$ \\
$\mathrm{RS}^{\mathrm{DDDH}}{ }^{\mathrm{f}}$ & 0.25 & $1 / \varepsilon_{\infty}^{\mathrm{sc}}$ & $\mu_{\mathrm{fit}}{ }^{\mathrm{g}}$ \\
$\mathrm{CAM}\left(0,1 / \varepsilon_{\infty}^{\mathrm{sc}}, \mu_{\mathrm{TF}}\right)^{\mathrm{a}}$ & 0 & $1 / \varepsilon_{\infty}^{\mathrm{sc}}$ & $\mu_{\mathrm{TF}}$ \\
\hline \hline
\end{tabular}

aThis work.

${ }^{\mathrm{b}}$ Reference [69].

${ }^{\mathrm{c}}$ Fitted $\mu$ parameter within a self-consistent loop.

${ }^{\mathrm{d}}$ Reference [68].

${ }^{\text {e}}$ Effective TF parameter within a self-consistent loop.

${ }^{\mathrm{f}}$ Reference [66].

${ }^{\mathrm{g}}$ Fitted $\mu$ parameter.

tional, namely, the long-range fraction of incorporated Fock exchange $\alpha_{\ell}$, is determined analogously to the global mixing parameter by setting $\alpha_{\ell}=1 / \varepsilon_{\infty}^{\mathrm{sc}}$. Finally, the inverse rangeseparation length $\mu$ is set to the Thomas-Fermi (TF) screening parameter

$$
\mu_{\mathrm{TF}}=\sqrt[6]{\frac{3 n_{v}}{\pi}},
$$

where $n_{v}$ is the valence-electron density [66]. For liquid water and hexagonal ice, Eq. (4) yields $\mu$ equal to 0.58 and $0.57 \mathrm{bohr}^{-1}$, respectively (cf. Sec. IV A). These values can be physically interpreted as the inverse of the $\mathrm{OH}$ bond length, $1 /(0.96 \AA) \approx 0.55 \mathrm{bohr}^{-1}$, and represent in this perspective a typical length scale of the considered material. The values of $\mu_{\mathrm{TF}}$ are also consistent with those obtained in Ref. [63]. We note that alternative definitions of the $\mu$ parameter relying on the effective TF screening [68], the Wigner-Seitz radius [66], or various fitting procedures $[66,69]$ have been suggested in the literature. However, it has been shown that these definitions yield almost identical $\mu$ values $[66,69,71]$ accompanied by insignificant effects on the band-gap estimates [66]. Therefore, we consider $\mu=\mu_{\mathrm{TF}}$ throughout this work. Overall, we investigate three range-separated DDH functionals in this study, denoted by $\operatorname{CAM}\left(1,1 / \varepsilon_{\infty}^{\mathrm{sc}}, \mu_{\mathrm{TF}}\right), \operatorname{CAM}\left(0.25,1 / \varepsilon_{\infty}^{\mathrm{sc}}, \mu_{\mathrm{TF}}\right)$, and $\operatorname{CAM}\left(0,1 / \varepsilon_{\infty}^{\mathrm{sc}}, \mu_{\mathrm{TF}}\right)$. We remark that the present functionals can be compared to similar range-separated DDH functionals in the literature, such as DD-RSH-CAM [69], DSH [68], and RS-DDH [66]. A comparison of these functionals is given in Table II. The differences are technical and originate from the definition of $\alpha_{\mathrm{s}}$ and the determination of the range-separation parameter $\mu$.

\section{Hybrid functionals satisfying Koopmans' condition}

The third class of advanced electronic-structure methods investigated in this work consists in hybrid functionals that 
TABLE III. Properties of the structural models used for liquid water $\ell-\mathrm{H}_{2} \mathrm{O}$ and hexagonal ice $\mathrm{Ih}$ : space group, lattice parameters $a$ and $c / a$, number $N$ of water molecules per unit cell, and mass density $\varrho$. The settings are taken from Refs. [8,128].

\begin{tabular}{lccccc}
\hline \hline Phase of water & Space group & $a(\AA)$ & $c / a$ & $N$ & $\varrho\left(\mathrm{g} / \mathrm{cm}^{3}\right)$ \\
\hline$\ell-\mathrm{H}_{2} \mathrm{O}$ & $P 1$ & 9.81 & & 32 & 1.01 \\
$\mathrm{Ih}$ & $P 6_{3} \mathrm{~cm}$ & 7.82 & 0.941 & 12 & 0.92 \\
\hline \hline
\end{tabular}

satisfy Koopmans' condition. This physical constraint states that within exact GKS theory [119] a single-particle energy level does not move upon electron occupation, thereby ensuring piecewise linearity of the total energy upon fractional electron occupation [57]. For approximate density functionals this constraint is generally violated, leading to the appearance of the many-body self-interaction error [126,127].

For the construction of hybrid functionals that satisfy Koopmans' condition, we apply the procedure outlined in Refs. $[75,78,80]$. First, we consider a supercell of the investigated material in which a point defect has been introduced. The associated single-particle energy level is then evaluated with a hybrid functional in the occupied and in the unoccupied charge state. The two calculations generally yield different defect levels due to the departure from Koopmans' condition. The energy difference between the single-particle levels in the two charge states is denoted by $\Delta_{\mathrm{K}}$. This deviation varies as a function of the undetermined parameter of the hybrid functional that we denote by $\alpha$. When the deviation vanishes for a specific value $\alpha_{\mathrm{K}}$, i.e., $\Delta_{\mathrm{K}}\left(\alpha_{\mathrm{K}}\right)=0$, Koopmans' condition is fulfilled. The hybrid functional defined by $\alpha_{\mathrm{K}}$ is then instrumental in obtaining a band-gap estimate for the host material. A graphical illustration of this procedure is given in Fig. 2 for the representative case of the $\mathrm{OH}$ radical in hexagonal ice. To ensure a consistent comparison with the DDH functionals, we construct hybrid functionals satisfying Koopmans' condition by considering both the global PBE0 and the range-separated CAM functionals.

\section{COMPUTATIONAL ASPECTS}

\section{A. Structural models}

The calculations carried out in this work make use of well-established structural models for liquid water [8] and hexagonal ice [128]. The properties of these models are summarized in Table III. Graphical illustrations are given in Fig. 3.

In the case of liquid water, we consider 20 snapshots taken from a molecular-dynamics simulation performed by Chen et al. [8,101]. The atomic structure in this simulation has been shown to be well equilibrated and to be consistent with the experimental radial distribution functions [8]. The adopted snapshots are evenly spaced in time and correspond to independent configurations of 32 water molecules at ambient temperature $(300 \mathrm{~K})$ [8]. The density of liquid water in the considered trajectory is only marginally higher than the experimental one [129] (by $\sim 1 \%$ ), which has been shown to negligibly affect the calculated band gaps [8]. Van der Waals interactions are incorporated in the water trajectory through the use of the revised Vydrow-Van Voorhis nonlocal density functional $[130,131]$. The empirical parameter $b$ has been set to 8.9, which ensures a realistic description of the structure of liquid water [8,132]. As discussed in Sec. II, we account for NQE through an a posteriori band-gap correction. It is therefore appropriate to consider configurations of liquid water in which the nuclei have been treated classically.

For hexagonal ice, we consider a unit cell as given in the Bernal-Fowler model [128]. This model comprises 12 water molecules arranged in a hexagonal lattice and has been adopted in several theoretical studies [34,51,132-134]. To ensure a consistent description of liquid water and hexagonal ice, we relaxed the atomic coordinates of the Bernal-Fowler model with the same revised Vydrow-Van Voorhis functional as used by Chen et al. [8]. The density of the original Bernal-Fowler model $\left(0.92 \mathrm{~g} / \mathrm{cm}^{3}\right)$ corresponds to that obtained at a temperature just below the melting point [128]. The density at this temperature differs only slightly from that obtained through extrapolation to $0 \mathrm{~K}$, namely, $0.93 \mathrm{~g} / \mathrm{cm}^{3}$ (see Refs. [50,52] and references therein). We checked that such an increase of the density leads to negligible changes in the calculated band gaps (less than $0.01 \mathrm{eV}$ at the PBE level of theory). Therefore, we adopt in this work the lattice constants (and thus the density) as originally proposed by Bernal and Fowler [128]. Furthermore, we remark that the present model carries a net dipole moment which is closely connected to the lattice-constant ratio $c / a$ [133]. To investigate the effect of this dipole, we modified this ratio such that the net dipole moment vanishes, but we found no relevant change in the band gap. Hence, we keep the unmodified $c / a$ ratio throughout this work.

We note that hexagonal ice is generally a proton-disordered phase of solid water. To account for this effect, various structural models have been proposed in the literature [135]. The most stable proton-ordered configuration of Ih is referred to as XIh [134]. It has been observed below the order-disorder transition temperature of $72 \mathrm{~K}$ [136] and exhibits the space group $C m c 2_{1}$ [137]. However, Engel et al. have shown that the band gaps calculated using the $C m c 2_{1}$ model and the Bernal-Fowler model differ by less than $0.02 \mathrm{eV}$ at the semilocal and hybrid-functional levels of theory [34]. Likewise, the computed band gaps for 16 distinct Ih models agree within $0.05 \mathrm{eV}$ when the zero-phonon renormalization is taken into account [34]. Therefore, it is justified to focus in our investigation on the Bernal-Fowler model as a representative structural configuration of Ih.

\section{B. Computational details}

The calculations presented in this work are carried out with norm-conserving pseudopotentials to describe core-valence interactions [138,139]. The energy cutoff for the plane-wave basis set is set at $85 \mathrm{Ry}$. The adopted $k$-point samplings for the bulk calculations in liquid water and hexagonal ice are $1 \times 1 \times 1$ (only the $\Gamma$ point) and $2 \times 2 \times 2$, respectively. We calculate the VBM of liquid water following the procedure outlined by Ambrosio et al. [11]. This implies the calculation of the average electron density of states (DOS) over the adopted selection of 20 water snapshots. The VBM is then determined through linear extrapolation of the valence-band 
wing in the DOS. This way of proceeding is convenient for two reasons. First, it leads to a fast convergence of the VBM with respect to the adopted supercell size $[8,11]$. Second, this procedure mimics the experimental determination of the VBM, which is based on an analogous extrapolation [6]. We note that the VBM obtained in this way is independent of the broadening, provided the smearing remains reasonably small (Gaussian broadening of $0.05 \mathrm{eV}$ in our work). For hexagonal ice, we determine the VBM through the highest occupied Kohn-Sham level. It is noteworthy that the VBM in hexagonal ice is located at the $X$ point resulting in an indirect band-gap transition $X \rightarrow \Gamma$. The direct band gap at the $\Gamma$ point is generally $\sim 0.1 \mathrm{eV}$ larger than the indirect one. The band gaps reported in the following correspond to the indirect transition.

The QSGW calculations in this work are performed with nonlocal commutators for the optical matrix element in the long-wavelength limit, as described in Ref. [140]. The contour deformation technique is used to evaluate the frequency dependence of the dielectric function [141]. We account for eight real and four imaginary frequencies. For the calculation of the dielectric function, we apply the formula of Adler [142] and Wiser [143] using an energy cutoff $E_{\text {cut }}^{\text {eps }}$ of 12 Ry and a total number $n_{\text {band }}$ of 2000 bands. In the QS $G W$ calculations, we self-consistently update only the lowest 600 bands and keep the higher-lying states unchanged as obtained with the semilocal PBE functional [100]. For the vertex corrections in the screening, we use the bootstrap exchange-correlation kernel of Sharma et al. [114] in the efficient head-only implementation of Chen et al. [8,38]. All QSGW calculations presented in this study are performed with the ABINIT software package [144].

In order to ensure the convergence of the $G W$ calculations, we separately extrapolate the band gap as a function of the cutoff $E_{\mathrm{cut}}^{\mathrm{eps}}$, the total number of bands $n_{\mathrm{band}}$, and the number of self-consistently updated bands. These extrapolations are performed through an exponential function of the energy cutoff and through linear functions of the inverse number of total bands and of the inverse number of updated bands [145]. For hexagonal ice, the extrapolations in $E_{\text {cut }}^{\text {eps }}$ and $n_{\text {band }}$ yield corrections of $\sim 0.08 \mathrm{eV}$, as illustrated in Fig. 4. The extrapolation in the number of updated bands gives a correction of only $0.01 \mathrm{eV}$. For liquid water, the respective corrections are $0.03,0.28$, and $0.13 \mathrm{eV}$. The corrections associated with the number of bands are larger than in the case of hexagonal ice, due to the larger simulation cell used for liquid water (cf. Sec. IV A). Hence, the QSGW band gaps are corrected by the sum of the three corrections determined above. This results in global corrections of 0.44 and $0.17 \mathrm{eV}$ for liquid water and hexagonal ice, respectively.

For the construction of DDH functionals, it is necessary to evaluate the high-frequency dielectric constant $\varepsilon_{\infty}$. To this end, we perform separate calculations using the finite electric field approach [146]. The proper convergence of $\varepsilon_{\infty}$ is ensured through a four-times-denser $k$-point sampling in the direction of the field. For hexagonal ice, we perform an isotropic average over the trace of the dielectric tensor to account for the small anisotropy in $\varepsilon_{\infty}[147,148]$.

The construction of hybrid functionals satisfying Koopmans' condition is based on single-particle levels of point

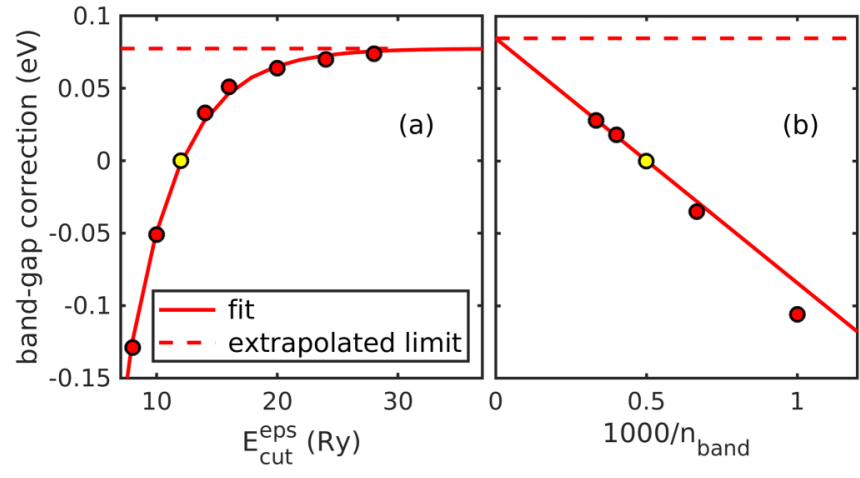

FIG. 4. Band-gap corrections (in eV) for hexagonal ice Ih according to the extrapolation of (a) the cutoff in the dielectric matrix $E_{\text {cut }}^{\text {eps }}$ and (b) the total number of bands $n_{\text {band }}$. The yellow circle indicates the parameter value generally used in the QSGW calculations and serves as reference for the band-gap corrections. The solid and the dashed line illustrate the fit and the extrapolated limit, respectively.

defects $[75,78,80]$. For liquid water, we generate such defects within the snapshots of Chen et al. [8]. For hexagonal ice, we generate the defects within a $2 \times 2 \times 2$ supercell based on the Bernal-Fowler model [128] (cf. Sec. IV A). For both systems, a $k$-point sampling at the sole $\Gamma$ point ensures converged single-particle levels. Spin-polarized defect calculations are performed whenever unpaired electrons occur. We note that no structural relaxation is allowed upon the creation of the point defects. This way of proceeding implies that the long-range screening is characterized entirely by the highfrequency dielectric constant $\varepsilon_{\infty}$. The spurious interactions with periodically repeated image charges is overcome through the use of accurate finite-size corrections to the single-particle levels $[149,150]$. To avoid systematic errors, we evaluate these corrections using dielectric constants that consistently correspond to the hybrid functionals used in the defect calculations.

All hybrid functional calculations in this work are performed with the QUANTUM-ESPRESSO software code [151]. We use the same numerical parameters and the same pseudopotentials [139] as in the calculations with ABINIT to ensure a meaningful comparison between the results of the two codes.

\section{BAND GAPS OF LIQUID WATER AND HEXAGONAL ICE}

\section{A. Quasiparticle self-consistent $G W$}

First, we examine the band gaps of liquid water and hexagonal ice as obtained with different flavors of the QSGW approach. In particular, we consider (i) the standard QSGW scheme and (ii) QSGE, which includes vertex corrections in the screening. The results of our calculations are shown in Table IV together with the experimental band-gap references.

The $G W$ schemes applied to liquid water and hexagonal ice give results that compare similarly with the corresponding experimental references. The standard QSGW method yields band gaps that are overestimated. Indeed, the calculated band gaps are higher by 0.4 and $0.1 \mathrm{eV}$ than the upper limit of the reference range for liquid water and hexagonal ice, respectively. Similar overestimations have been observed for various other materials $[37,38]$ and have been attributed to the neglect 
TABLE IV. Fundamental band gaps (in eV) for liquid water $\ell-\mathrm{H}_{2} \mathrm{O}$ and hexagonal ice Ih as calculated with the QSGW and QS $G \tilde{W}$ methods. The band gaps correspond to temperatures of 300 and $77 \mathrm{~K}$, respectively. The reported band gaps include the corrections associated with NQE and ZPR (cf. Sec. II). Band gaps inferred from experimental data are given as reference (cf. Sec. II).

\begin{tabular}{lccc}
\hline \hline Phase of water & QSGW & QSGW & Expt. \\
\hline$\ell-\mathrm{H}_{2} \mathrm{O}$ & 9.6 & 9.1 & $9.0 \pm 0.2^{\mathrm{a}}$ \\
Ih & 9.8 & 9.3 & $9.4 \pm 0.3^{\mathrm{b}}$
\end{tabular}

${ }^{\mathrm{a}} T=300 \mathrm{~K}$

${ }^{\mathrm{b}} T=77 \mathrm{~K}$.

of electron-hole interactions in the calculation of the screened $W$. We overcome this limitation by carrying out QSG $\tilde{W}$ calculations, which include vertex corrections in the screening $(\tilde{W})$. In this scheme, the band gaps are smaller by $0.5 \mathrm{eV}$ with respect to those obtained without vertex corrections. When comparing to experimental values, we record very good agreement for both phases of water since the calculated band gaps fall within the reference range. Such an accuracy is in agreement with previous studies, which have found typical mean absolute errors of $\sim 0.2 \mathrm{eV}$ for such QS $G \tilde{W}$ calculations [37,38,118].

We note that our QSGE $\tilde{W}$ calculations can be confronted with other $G W$ studies in the literature. For liquid water, several one-shot $G_{0} W_{0}$ calculations have been performed. With respect to our QS $G \tilde{W}$ scheme, this method systematically yields underestimated band gaps when semilocal results are used as starting points $[7,39,40,44]$ and overestimated band gaps when hybrid functional results are used [9]. As far as previous self-consistent $G W$ methods are concerned, we take into consideration the recent study of Chen et al. [8]. These authors obtained a band gap of $8.9 \mathrm{eV}$ also by means of QSGW $\tilde{W}$ calculations. The difference of $0.2 \mathrm{eV}$ with respect to the present results should be attributed to small differences in the computational setup. Indeed, we evaluate the nonlocal commutators for the optical matrix element in the long-wavelength limit as described in Ref. [140] and use more stringent convergence criteria to overcome the criticism raised in Ref. [41]. The latter work reported a band gap of liquid water obtained with the evGW scheme. The calculated band gap of $9.07 \mathrm{eV}$ is in good agreement with the present QSGW band gap, despite the absence of explicit vertex corrections in the evGW approach [41]. Our results can also be compared with those of Kharche et al. [16]. By means of full-frequencyspectrum-only self-consistent $G W$ calculations, these authors calculated a band gap of $9.53 \mathrm{eV}$ for classical water, which was later revised to $9.7 \mathrm{eV}$ [16]. Accounting for a band-gap reduction of $0.7 \mathrm{eV}$ due to NQE [8], we correct their band-gap estimate to $9.0 \mathrm{eV}$. This result has been obtained without including explicit vertex corrections, but is nevertheless in agreement with our QSG $\tilde{W}$ band gap of $9.1 \mathrm{eV}$.

For hexagonal ice, fewer $G W$ band-gap calculations can be found in the literature. Yim et al. reported a band gap of $8.9 \mathrm{eV}$ obtained with one-shot $G_{0} W_{0}$ calculations. Similarly, Fang et al. found a band gap of $9.17 \mathrm{eV}$ by means of a partially self-consistent $G W_{0}$ approach [43]. However, both studies did not account for the sizable zero-phonon renormalization of
TABLE V. High-frequency dielectric constant $\varepsilon_{\infty}^{\text {sc }}$ of liquid water $\ell-\mathrm{H}_{2} \mathrm{O}$ and hexagonal ice $\mathrm{Ih}$ as calculated self-consistently within the DDH procedure (cf. Sec. III B). The dielectric constants are obtained either with the global functional $\operatorname{PBE0}\left(1 / \varepsilon_{\infty}^{\mathrm{sc}}\right)$ or with the range-separated functional $\operatorname{CAM}\left(\alpha_{\mathrm{s}}, 1 / \varepsilon_{\infty}^{\mathrm{sc}}, \mu_{\mathrm{TF}}\right)$. Various settings for the short-range fraction of Fock exchange $\alpha_{\mathrm{s}}$ are distinguished. Experimental dielectric constants are given for comparison.

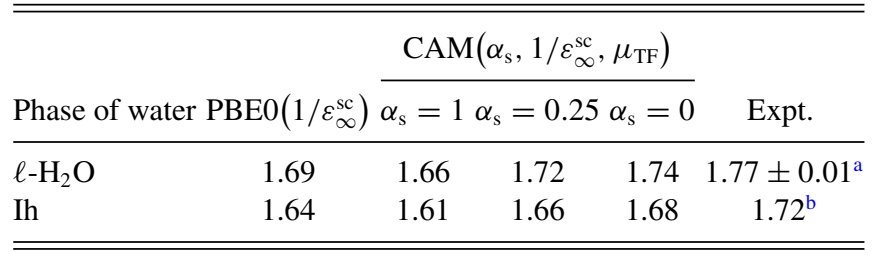

${ }^{a}$ References [81,83].

${ }^{\mathrm{b}}$ Reference [152].

$\sim 1.5 \mathrm{eV}$ (cf. Sec. II). Therefore, the apparent agreement with our QSGW $\tilde{W}$ calculations results from error cancellation. In another investigation, Hahn et al. determined a larger band gap of $\sim 10 \mathrm{eV}$ using an approximate $G W$ scheme, which incorporates a model dielectric function and lacks self-consistency [42]. When this result is corrected for ZPR, one finds a band gap of $\sim 8.5 \mathrm{eV}$, to be compared with the band gap of $9.3 \mathrm{eV}$ obtained in the present work.

Overall, we conclude that the QS $G \tilde{W}$ approach used in the present work corresponds to the most elaborate theoretical framework applied so far to either liquid water or hexagonal ice. Thus, this scheme is expected to give the most accurate estimates for the band gaps of these systems.

\section{B. Dielectric-dependent hybrid functionals}

We here devote special attention to DDH functionals and to their band-gap estimates for liquid water and hexagonal ice. The free parameters of these functionals are determined through the dielectric response of the material under investigation (cf. Sec. III B). In this section, we therefore present the band gaps resulting from the DDH functionals together with their corresponding dielectric constants.

First, we apply the DDH procedure to the global hybrid functional $\operatorname{PBE} 0(\alpha)$. The dielectric constant as calculated within the self-consistent workflow is shown in Fig. 5(a) for the case of hexagonal ice. We observe that $\varepsilon_{\infty}$ converges within four iterations to the self-consistent value $\varepsilon_{\infty}^{\mathrm{sc}}$. It is noteworthy that this specific value is independent of the considered starting point [63], as we checked using two distinct initializations, namely, from results obtained with the functionals PBE and PBE0(0.7) (cf. Fig. 5). The calculated value of $\varepsilon_{\infty}^{\mathrm{sc}}$ is in agreement with its experimental counterpart showing an underestimation of only $\sim 5 \%$ (Table V). For liquid water, the self-consistent calculation of the dielectric constant proceeds analogously and results in a similar comparison with experiment (Table V). In this case, $\varepsilon_{\infty}^{\text {sc }}$ is only marginally affected by the considered water snapshot. We analyzed various snapshots and observed variations in $\varepsilon_{\infty}^{\mathrm{sc}}$ smaller than $\sim 0.02$. It is sufficient to average over five different water snapshots to achieve a converged mean value with a standard deviation of less than 0.01 . 


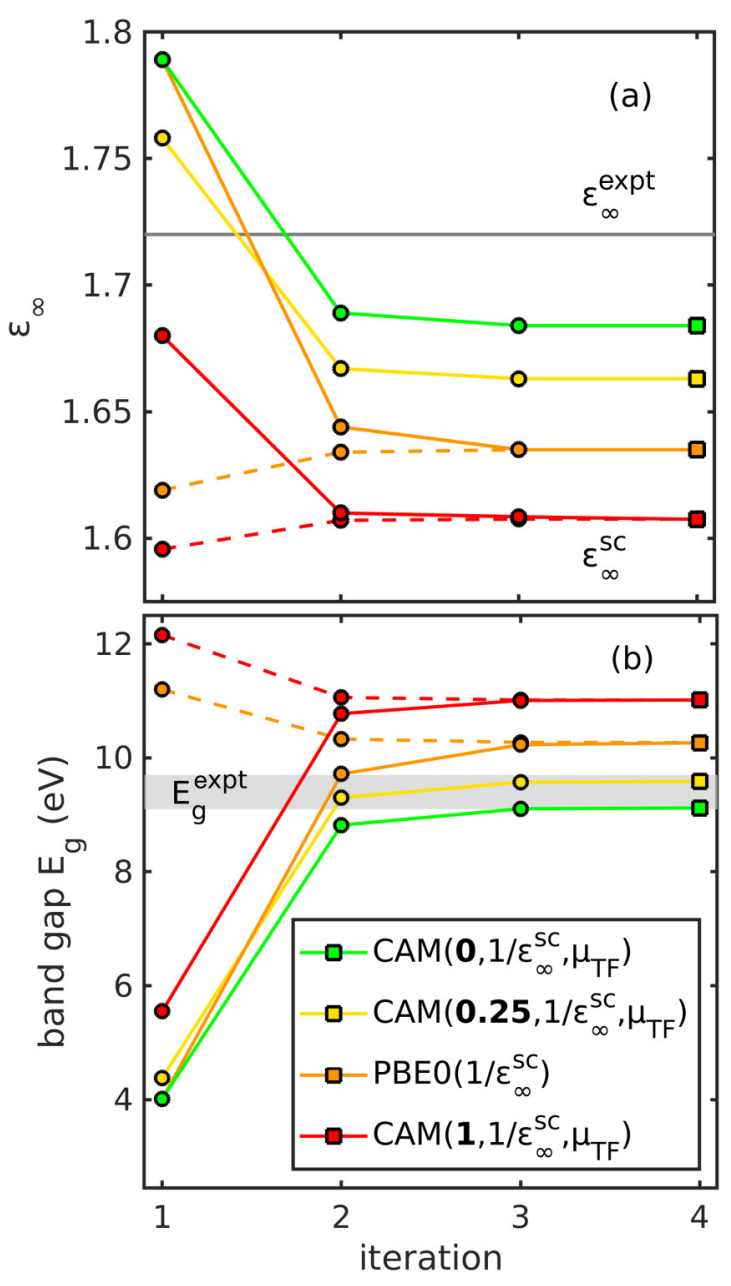

FIG. 5. Convergence of (a) the dielectric constant $\varepsilon_{\infty}$ and (b) the band gap $E_{g}$ within the self-consistent DDH scheme for hexagonal ice Ih. Iteration steps and converged results are visualized as circles and squares, respectively. The global hybrid functional $\operatorname{PBE} 0\left(1 / \varepsilon_{\infty}^{\mathrm{sc}}\right)$ and the range-separated hybrid functionals $\operatorname{CAM}\left(0,1 / \varepsilon_{\infty}^{\mathrm{sc}}, \mu_{\mathrm{TF}}\right)$, $\operatorname{CAM}\left(0.25,1 / \varepsilon_{\infty}^{\mathrm{sc}}, \mu_{\mathrm{TF}}\right)$, and $\operatorname{CAM}\left(1,1 / \varepsilon_{\infty}^{\mathrm{sc}}, \mu_{\mathrm{TF}}\right)$ are examined. The independence of the converged results from the starting point is illustrated through distinct initializations: The global $\operatorname{PBE} 0\left(1 / \varepsilon_{\infty}^{\mathrm{sc}}\right)$ functional has been started from either the PBE (solid orange line) or the PBE0(0.7) (orange dashed line) functional, while the rangeseparated $\operatorname{CAM}\left(1,1 / \varepsilon_{\infty}^{\mathrm{sc}}, \mu_{\mathrm{TF}}\right)$ functional has been started from either the $\operatorname{CAM}\left(1,0, \mu_{\mathrm{TF}}\right)$ (red solid line) or the $\operatorname{CAM}\left(1,0.7, \mu_{\mathrm{TF}}\right)$ (red dashed line) functional. The reported band gaps include the corrections due to ZPR (cf. Sec. II). The horizontal gray line corresponds to the experimental high-frequency dielectric constant $\varepsilon_{\infty}^{\text {expt }}$ [152]. The shaded area indicates the interval corresponding to the experimental reference values $E_{g}^{\text {expt }}$ inferred in Sec. II.

Next we consider the band gaps as obtained with the global $\mathrm{DDH}$ functional. The convergence of the band gap within the DDH procedure is shown in Fig. 5(b) for the case of hexagonal ice. Analogously to the dielectric constant, the band gap converges within four iterations irrespective of the adopted starting point. The converged result for the band gap corresponds to the functional $\operatorname{PBE} 0\left(1 / \varepsilon_{\infty}^{\mathrm{sc}}\right)$. The convergence behavior for liquid water is analogous to that of hexagonal ice shown in Fig. 5(b). The spread of 0.01 in $\varepsilon_{\infty}^{\text {sc }}$ due to
TABLE VI. Fundamental band gaps (in $\mathrm{eV}$ ) of liquid water $\ell-\mathrm{H}_{2} \mathrm{O}$ and hexagonal ice $\mathrm{Ih}$ as calculated with DDH functionals. The band gaps correspond to temperatures of 300 and $77 \mathrm{~K}$, respectively. The band gaps are obtained either with the global functional $\operatorname{PBE} 0\left(1 / \varepsilon_{\infty}^{\mathrm{sc}}\right)$ or with the range-separated functional $\operatorname{CAM}\left(\alpha_{\mathrm{s}}, 1 / \varepsilon_{\infty}^{\mathrm{sc}}, \mu_{\mathrm{TF}}\right)$. The value of the parameter $1 / \varepsilon_{\infty}^{\mathrm{sc}}$ is given in parentheses. Various settings for the short-range fraction of Fock exchange $\alpha_{\mathrm{s}}$ are distinguished. The reported band gaps include the corrections associated with NQE and ZPR (cf. Sec. II). Band gaps inferred from experimental data are given as reference (cf. Sec. II).

\begin{tabular}{lccccc}
\hline \hline & & \multicolumn{3}{c}{$\operatorname{CAM}\left(\alpha_{\mathrm{s}}, 1 / \varepsilon_{\infty}^{\mathrm{sc}}, \mu_{\mathrm{TF}}\right)$} & \\
\cline { 3 - 5 } Phase of water $\operatorname{PBE0}\left(1 / \varepsilon_{\infty}^{\mathrm{sc}}\right)$ & $\alpha_{\mathrm{s}}=1$ & $\alpha_{\mathrm{s}}=0.25$ & $\alpha_{\mathrm{s}}=0$ & Expt. \\
\hline$\ell-\mathrm{H}_{2} \mathrm{O}$ & 10.0 & 10.9 & 9.5 & 9.0 & $9.0 \pm 0.2^{\mathrm{a}}$ \\
& $(0.59)$ & $(0.60)$ & $(0.58)$ & $(0.57)$ & \\
$\mathrm{Ih}$ & 10.3 & 11.0 & 9.6 & 9.1 & $9.4 \pm 0.3^{\mathrm{b}}$ \\
& $(0.61)$ & $(0.62)$ & $(0.60)$ & $(0.59)$ & \\
\hline \hline
\end{tabular}

${ }^{\mathrm{a}} T=300 \mathrm{~K}$.

${ }^{\mathrm{b}} T=77 \mathrm{~K}$.

the statistical variety of water snapshots results in variations smaller than $0.1 \mathrm{eV}$ in the band-gap estimate.

The band gaps obtained with the $\operatorname{PBE} 0\left(1 / \varepsilon_{\infty}^{\mathrm{sc}}\right)$ functional for both liquid water and hexagonal ice are given in Table VI together with experimental references. We observe an overestimation of the band gap for both water phases. With respect to the upper limit of the reference interval, the deviations amount to 0.8 and $0.6 \mathrm{eV}$ for liquid water and hexagonal ice, respectively. We attribute this band-gap overestimation to the underestimation of the dielectric constant with respect to the experimental value [cf. Fig. 5(a) and Table V].

Our result for the band gap of hexagonal ice can be compared with that obtained by Skone et al., who used a similar self-consistent approach [63]. These authors reported a band gap of $11.71 \mathrm{eV}$ for hexagonal ice [63]. When this result is corrected for the ZPR, one obtains a band gap of $10.2 \mathrm{eV}$, in good agreement with the value of $10.3 \mathrm{eV}$ obtained in the present work.

In the second part of this section, we apply the DDH construction procedure to the range-separated functional $\operatorname{CAM}\left(\alpha_{\mathrm{s}}, \alpha_{\ell}, \mu\right)$. This functional incorporates different fractions of Fock exchange in the short $\left(\alpha_{\mathrm{s}}\right)$ and in the long range $\left(\alpha_{\ell}\right)$. The transition between the two limiting regimes is mediated by the range-separation parameter $\mu$. For the determination of these parameters, we proceed as discussed in Sec. III B. In particular, we consider three different settings for the short-range fraction of Fock exchange $\alpha_{\mathrm{s}}$, namely, $\alpha_{\mathrm{s}}$ equal to $1,0.25$, and 0 . The respective range-separated DDH functionals are then denoted by $\operatorname{CAM}\left(1,1 / \varepsilon_{\infty}^{\mathrm{sc}}, \mu_{\mathrm{TF}}\right)$, $\operatorname{CAM}\left(0.25,1 / \varepsilon_{\infty}^{\mathrm{sc}}, \mu_{\mathrm{TF}}\right)$, and $\operatorname{CAM}\left(0,1 / \varepsilon_{\infty}^{\mathrm{sc}}, \mu_{\mathrm{TF}}\right)$.

The convergence of the DDH scheme for the three functionals considered is shown in Fig. 5 in the case of hexagonal ice. The convergence behavior is generally very similar to that of the global DDH functional. More specifically, convergence is similarly achieved within four iterations irrespective of the adopted starting point (cf. Fig. 5). This observation underlines the remarkable numerical stability of the DDH scheme in accord with previous studies in the literature $[63,66,68,69]$. 
However, the results obtained with different settings of the parameter $\alpha_{\mathrm{s}}$ exhibit differences that deserve close attention. First, we focus on the self-consistently determined dielectric constants $\varepsilon_{\infty}^{\mathrm{sc}}$. The values resulting from the three rangeseparated DDH functionals are given in Table $\mathrm{V}$ for both liquid water and hexagonal ice. We observe a systematic variation of $\varepsilon_{\infty}^{\mathrm{sc}}$ as a function of $\alpha_{\mathrm{s}}$. More specifically, the smaller the short-range Fock exchange $\alpha_{\mathrm{s}}$, the higher the dielectric constant $\varepsilon_{\infty}^{\text {sc }}$. This systematic trend manifests in deviations from the experimental references ranging from only $\sim 2 \%$ for $\alpha_{\mathrm{s}}=0$ up to $\sim 6 \%$ for $\alpha_{\mathrm{s}}=1$. Overall, these dielectric constants agree with experiment irrespective of $\alpha_{\mathrm{s}}$. However, the best agreement is clearly achieved for $\alpha_{\mathrm{s}}=0$ and the corresponding functional $\operatorname{CAM}\left(0,1 / \varepsilon_{\infty}^{\mathrm{sc}}, \mu_{\mathrm{TF}}\right)$.

Next we investigate the band gaps resulting from the three range-separated DDH functionals. Our results for liquid water and hexagonal ice are given in Table VI. We find that also the band gap exhibits a systematic variation according to the value set for the parameter $\alpha_{\mathrm{s}}$. More specifically, the smaller the short-range Fock exchange $\alpha_{\mathrm{s}}$, the smaller the band gap obtained with the corresponding DDH functional $\operatorname{CAM}\left(\alpha_{\mathrm{s}}, 1 / \varepsilon_{\infty}^{\mathrm{sc}}, \mu_{\mathrm{TF}}\right)$. In the following, we separately discuss the results for the three settings of $\alpha_{\mathrm{s}}$.

For $\alpha_{\mathrm{s}}=1$ and the associated DDH functional $\operatorname{CAM}\left(1,1 / \varepsilon_{\infty}^{\mathrm{sc}}, \mu_{\mathrm{TF}}\right)$, we obtain strongly overestimated band gaps. With respect to the upper limit of the reference interval, the deviations amount to 1.7 and $1.3 \mathrm{eV}$ for liquid water and hexagonal ice, respectively. This result is unexpected insofar as the similar DD-RSH-CAM approach of Chen et al. provides accurate band gaps for a variety of semiconductors and insulators [69]. Similarly, good agreement with experiment has been found for a series of metal-halide perovskites [80]. The origin of the failure of this method for liquid water and hexagonal ice remains unclear at the moment and deserves further investigation.

For $\alpha_{\mathrm{s}}=0.25$ and the corresponding $\mathrm{DDH}$ functional $\operatorname{CAM}\left(0.25,1 / \varepsilon_{\infty}^{\mathrm{sc}}, \mu_{\mathrm{TF}}\right)$, we observe good agreement with the experimental references. Indeed, for hexagonal ice the computed band gap falls within the reference interval. For liquid water, we observe a small overestimation of $0.3 \mathrm{eV}$ with respect to the upper limit of the range of experimental values. Interestingly, the band gaps obtained with $\operatorname{CAM}\left(0.25,1 / \varepsilon_{\infty}^{\mathrm{sc}}, \mu_{\mathrm{TF}}\right)$ can be confronted with the similar RS-DDH approach of Skone et al. [66]. These authors reported a band gap for hexagonal ice of $10.94 \mathrm{eV}$ [66]. When this result is corrected for $\mathrm{ZPR}$, one finds a band gap of $9.4 \mathrm{eV}$, in good agreement with the value of $9.6 \mathrm{eV}$ found in the present work.

For $\alpha_{\mathrm{s}}=0$ and the associated DDH functional $\operatorname{CAM}\left(0,1 / \varepsilon_{\infty}^{\mathrm{sc}}, \mu_{\mathrm{TF}}\right)$, we achieve the highest accuracy among the examined range-separated DDH functionals. Indeed, we find band-gap estimates falling within the experimental range for both considered phases of water.

On the basis of the agreement with experiment for the dielectric constant, we are led to elect the $\operatorname{CAM}\left(0,1 / \varepsilon_{\infty}^{\mathrm{sc}}, \mu_{\mathrm{TF}}\right)$ as the most reliable range-separated DDH functional for liquid water and hexagonal ice. This assessment is further supported by the band gaps achieved with this functional, which fall within the range of experimental values for the two phases of water.
TABLE VII. Fundamental band gap (in eV) of hexagonal ice Ih as calculated with range-separated DDH functionals of the form $\operatorname{CAM}\left(\alpha_{\mathrm{s}}, 1 / \varepsilon_{\infty}^{\mathrm{sc}}, \mu\right)$. Two values for the range-separation parameter $\mu$ are considered: $\mu_{\mathrm{WS}}=0.53 \mathrm{bohr}^{-1}$ and $\mu_{\mathrm{TF}}=0.57 \mathrm{bohr}^{-1}$. The short-range fraction of Fock exchange $\alpha_{\mathrm{s}}$ is set to $1,0.25$, or 0 . The value of the self-consistently determined dielectric constant $\varepsilon_{\infty}^{\text {sc }}$ is given in parentheses. The reported band gaps include the correction associated with the ZPR (cf. Sec. II).

\begin{tabular}{lccc}
\hline \hline$\mu$ & $\alpha_{\mathrm{s}}=1$ & $\alpha_{\mathrm{s}}=0.25$ & $\alpha_{\mathrm{s}}=0$ \\
\hline$\mu_{\mathrm{WS}}$ & $11.1(1.60)$ & $9.5(1.67)$ & $9.0(1.69)$ \\
$\mu_{\mathrm{TF}}$ & $11.0(1.61)$ & $9.6(1.66)$ & $9.1(1.68)$ \\
\hline \hline
\end{tabular}

The various range-separated DDH functionals investigated in this work yield differing results both for liquid water and for hexagonal ice. More specifically, the different settings of the parameter $\alpha_{\mathrm{s}}$ result in notable variations in the self-consistently determined dielectric constant $\varepsilon_{\infty}^{\mathrm{sc}}(\sim 0.1)$ as well as in the band-gap estimate $E_{\mathrm{g}}(\sim 2 \mathrm{eV})$. These sizable variations raise the question to what extent these results are affected by setting the range-separation parameter $\mu$ to $\mu_{\mathrm{TF}}$. To address this issue, we construct range-separated DDH functionals using a different setting for $\mu$, namely, the WignerSeitz screening parameter $\mu_{\mathrm{WS}}$ as defined in Ref. [66]. The results of these calculations are given in Table VII. We observe that the distinct settings of the range-separation parameter $\mu$ affect $\varepsilon_{\infty}^{\mathrm{sc}}$ only by $\sim 0.01$. These changes in the dielectric constant are accompanied by variations in the band-gap estimates of $\sim 0.1 \mathrm{eV}$. Therefore, we conclude that setting the range-separation parameter $\mu$ to $\mu_{\mathrm{TF}}$ does not critically affect the performance of the range-separated DDH functionals. The observed variations among these functionals can thus be attributed predominantly to the different settings of the short-range fraction of Fock exchange $\alpha_{\mathrm{s}}$.

Before closing this section, we question to what extent the nature of the present DDH functionals can be considered nonempirical. The global hybrid functional $\operatorname{PBE} 0\left(1 / \varepsilon_{\infty}^{\mathrm{sc}}\right)$ is entirely determined through the dielectric response of the material and is therefore fully nonempirical. However, for the range-separated functionals, the specific value adopted for $\alpha_{\mathrm{s}}$ has a significant effect on the resulting band gaps. In a fully nonempirical DDH scheme, these different settings should be either irrelevant for the outcome or determined on the basis of an exact property of generalized Kohn-Sham theory [119]. This is manifestly not the case for the range-separated DDH functionals. This remaining indetermination also underlies the lack of consistency among the various range-separated DDH approaches in the literature $[66,68,69]$. Despite this open issue, we consider the functional $\operatorname{CAM}\left(0,1 / \varepsilon_{\infty}^{\mathrm{sc}}, \mu_{\mathrm{TF}}\right)$ as the most reliable DDH functional on the grounds that it achieves the best agreement with the experimental dielectric constants.

\section{Hybrid functionals satisfying Koopmans' condition}

We now focus on hybrid functionals satisfying Koopmans' condition. For the construction of such functionals, we apply the procedure outlined in Sec. III C to various localized states in the two investigated phases of water. We introduce such states through interstitial atoms $\left(\mathrm{H}_{\mathrm{i}}, \mathrm{F}_{\mathrm{i}}, \mathrm{Cl}_{\mathrm{i}}\right.$, and $\left.\mathrm{Br}_{\mathrm{i}}\right)$, 

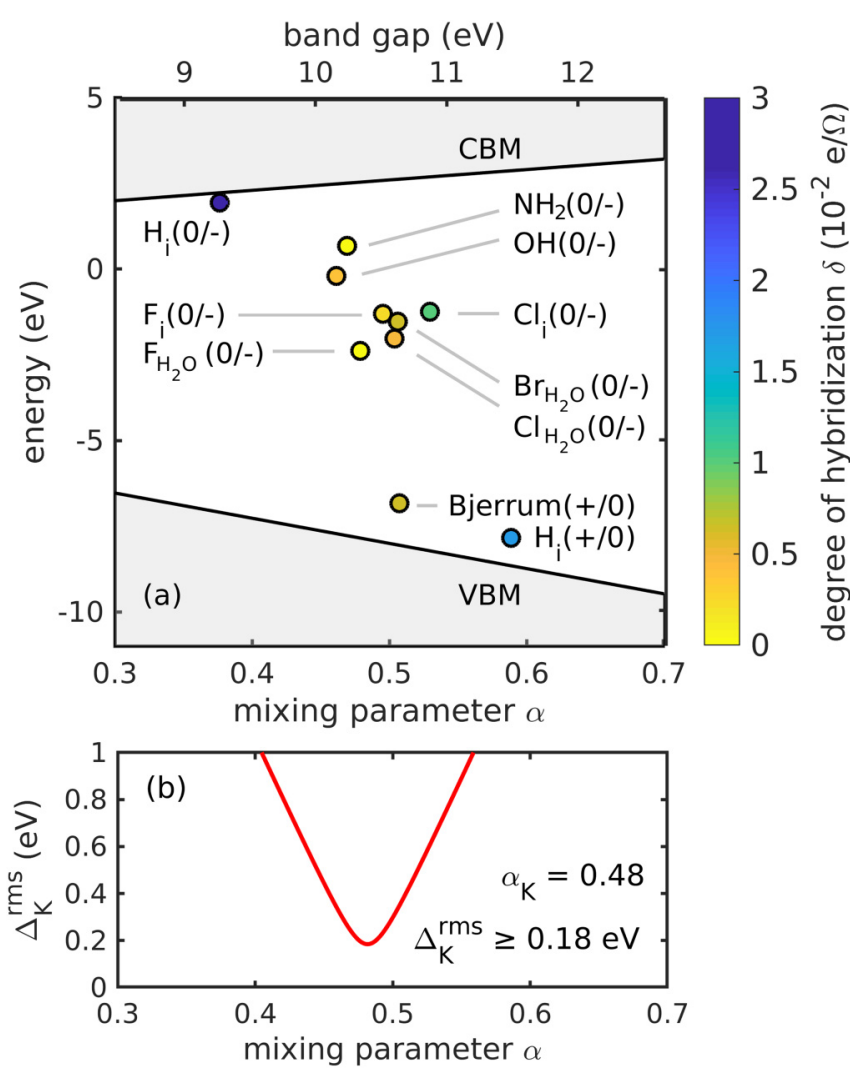

FIG. 6. (a) Band edges and defect energies for various localized states in hexagonal ice displayed versus the mixing parameter $\alpha$ incorporated in the $\operatorname{PBE} 0(\alpha)$ functional. The degree of hybridization $\delta$ of each localized state is visualized through the color scale. The indicated band gaps are obtained with the functional $\operatorname{PBEO}(\alpha)$ prior to the application of the corrections described in Sec. II. (b) The rms deviation from Koopmans' condition $\Delta_{\mathrm{K}}^{\mathrm{rms}}$ as a function of the mixing parameter $\alpha$. The value of $\alpha_{\mathrm{K}}$ and the corresponding minimum of $\Delta_{\mathrm{K}}^{\mathrm{rms}}$ are indicated.

substitutional atoms $\left(\mathrm{F}_{\mathrm{H}_{2} \mathrm{O}}, \mathrm{Cl}_{\mathrm{H}_{2} \mathrm{O}}\right.$, and $\left.\mathrm{Br}_{\mathrm{H}_{2} \mathrm{O}}\right)$, or inorganic radicals $\left(\mathrm{OH}\right.$ and $\left.\mathrm{NH}_{2}\right)$. In the case of hexagonal ice, we also consider the Bjerrum defect pair, which results from locally disregarding the second ice rule [153-155]. More specifically, one water molecule is pivoted in such a way that one pair of neighboring oxygen atoms is formed with no intermediate hydrogen atom and another pair occurs with two intermediate hydrogen atoms [155]. For each considered localized state, the degree of hybridization $\delta$ is computed [78]. This quantity measures the undesired hybridization between the localized defect state and the delocalized band-edge states. Through the minimization of $\delta$, we can identify the most reliable localized defect states $[78,80,156]$.

First, we examine the global hybrid functional $\operatorname{PBE} 0(\alpha)$ and determine the mixing parameter $\alpha_{\mathrm{K}}$ which satisfies Koopmans' condition. Our results are shown in Fig. 6(a) for the case of hexagonal ice. We find different values of $\alpha_{\mathrm{K}}$ for the various localized states. The $+/ 0$ and $0 /-$ charge transitions of the hydrogen interstitial $\mathrm{H}_{\mathrm{i}}$ determine the maximal and minimal values of $\alpha_{\mathrm{K}}$. Their sizable variation can be attributed to strong hybridizations with the band-edge states. The mixing parameters obtained through the other localized states fall within a much narrower range. This property goes together with a systematically lower degree of hybridization for the involved defects. The smallest $\delta$ are observed for the radical $\mathrm{OH}$, the radical $\mathrm{NH}_{2}$, the fluorine interstitial $\mathrm{F}_{\mathrm{i}}$, the fluorine substitutional $\mathrm{F}_{\mathrm{H}_{2} \mathrm{O}}$, and the chlorine substitutional $\mathrm{Cl}_{\mathrm{H}_{2} \mathrm{O}}$. The Bjerrum defect pair, the bromine substitutional $\mathrm{Br}_{\mathrm{H}_{2} \mathrm{O}}$, and chlorine interstitial $\mathrm{Cl}_{\mathrm{i}}$ also show rather small values of $\delta$, but yet noticeably larger than the previous five defects.

To account for the different behaviors of the various localized states, we proceed in the following way. We consider the five defects with the lowest degree of hybridization and compute the root mean square (rms) of their individual deviations from Koopmans' condition through

$$
\Delta_{\mathrm{K}}^{\mathrm{rms}}(\alpha)=\sqrt{\frac{1}{5} \sum_{i=1}^{5}\left[\Delta_{\mathrm{K}}^{i}(\alpha)\right]^{2}} .
$$

The rms deviation $\Delta_{\mathrm{K}}^{\mathrm{rms}}$ resulting from Eq. (5) is displayed in Fig. 6(b) for the case of hexagonal ice. We find that $\Delta_{\mathrm{K}}^{\mathrm{rms}}$ depends continuously on the fraction of Fock exchange $\alpha$ and reaches a minimum for a specific value in close proximity to the crossing points of the individual defects. Hence, this minimum accounts effectively for the various localized states and enables us to determine $\alpha_{\mathrm{K}}$ in a robust fashion. The minimum value of $\Delta_{\mathrm{K}}^{\mathrm{rms}}$ is found to be $\sim 0.2 \mathrm{eV}$, which corresponds to $\sim 2 \%$ of the band gaps involved. This indicates that Koopmans' condition is effectively fulfilled for the various localized states, albeit with a finite accuracy. We checked that the inclusion of the more hybridized bromine substitutional $\mathrm{Br}_{\mathrm{H}_{2} \mathrm{O}}$ and Bjerrum defect pair among the defects considered in Eq. (5) affects the extracted $\alpha_{\mathrm{K}}$ by less than 0.01, corresponding to changes of less than $0.1 \mathrm{eV}$ in the band-gap estimate. We therefore consider only the five defects with the lowest $\delta$. With the procedure outlined above, we find mixing parameters $\alpha_{\mathrm{K}}$ of 0.47 and 0.48 for liquid water and hexagonal ice, respectively. In particular, the result for liquid water is in good agreement with the value of $\alpha=0.45$ determined empirically by Ambrosio et al. [10]. In the case of liquid water, $\alpha_{\mathrm{K}}$ is found to be almost independent of the considered water snapshot, with variations within $\sim 0.01$. Therefore, it is sufficient to average over five different water snapshots to achieve a converged mean value.

The band gaps obtained with the functional $\operatorname{PBE} 0\left(\alpha_{\mathrm{K}}\right)$ are compared with the experimental reference values in Table VIII. The corresponding mixing parameter $\alpha_{\mathrm{K}}$ are given in parentheses. For liquid water, we observe a small band-gap underestimation of $0.1 \mathrm{eV}$ with respect to the lower bound of the experimental range. Similarly, we find an underestimation of $0.2 \mathrm{eV}$ for hexagonal ice. This level of accuracy is in line with previous studies employing the nonempirical functional $\operatorname{PBEO}\left(\alpha_{\mathrm{K}}\right)$ for band-gap predictions $[75,78,80]$.

It is of interest to compare the present results obtained with the functional $\operatorname{PBEO}\left(\alpha_{\mathrm{K}}\right)$ with those obtained with the functional $\operatorname{PBE} 0\left(1 / \varepsilon_{\infty}^{\mathrm{sc}}\right)$ (cf. Table VI). Both originate from the global hybrid functional $\operatorname{PBEO}(\alpha)$, but the parameters are determined through distinct construction schemes. We find that the mixing parameter $1 / \varepsilon_{\infty}^{\mathrm{sc}}$ is systematically higher than $\alpha_{\mathrm{K}}$. Consequently, the same holds for the respective band gaps obtained with $\operatorname{PBE} 0\left(1 / \varepsilon_{\infty}^{\mathrm{sc}}\right)$ and $\operatorname{PBE} 0\left(\alpha_{\mathrm{K}}\right)$. With 
TABLE VIII. Fundamental band gaps (in eV) of liquid water $\ell-\mathrm{H}_{2} \mathrm{O}$ and hexagonal ice $\mathrm{Ih}$ as calculated with hybrid functionals satisfying Koopmans' condition. The band gaps correspond to temperatures of 300 and $77 \mathrm{~K}$, respectively. The band gaps are obtained either with the global functional $\operatorname{PBE} 0\left(\alpha_{\mathrm{K}}\right)$ or with the range-separated functional $\operatorname{CAM}\left(\alpha_{\mathrm{s}, \mathrm{K}}, 1 / \varepsilon_{\infty}^{\text {expt }}, \mu_{\mathrm{TF}}\right)$. The values of the parameters $\alpha_{\mathrm{K}}$ and $\alpha_{\mathrm{s}, \mathrm{K}}$ are given in parentheses. The reported band gaps include the corrections associated with NQE and ZPR (cf. Sec. II). Band gaps inferred from experimental data are given as reference (cf. Sec. II).

\begin{tabular}{lccc}
\hline \hline Phase of water & $\operatorname{PBE0}\left(\alpha_{\mathrm{K}}\right)$ & $\operatorname{CAM}\left(\alpha_{\mathrm{s}, \mathrm{K}}, 1 / \varepsilon_{\infty}^{\text {expt }}, \mu_{\mathrm{TF}}\right)$ & Expt. \\
\hline$\ell-\mathrm{H}_{2} \mathrm{O}$ & $8.7(0.47)$ & $9.2(0.18)$ & $9.0 \pm 0.2^{\mathrm{a}}$ \\
Ih & $8.9(0.48)$ & $9.3(0.18)$ & $9.4 \pm 0.3^{\mathrm{b}}$
\end{tabular}

${ }^{\mathrm{a}} T=300 \mathrm{~K}$.

${ }^{\mathrm{b}} T=77 \mathrm{~K}$.

respect to the experimental band-gap references, $\operatorname{PBE} 0\left(\alpha_{\mathrm{K}}\right)$ shows a significantly better accord than $\operatorname{PBE} 0\left(1 / \varepsilon_{\infty}^{\mathrm{sc}}\right)$. We note that similar considerations have been found to apply to the metal-halide perovskites studied in Ref. [80]. While both Koopmans' condition and the asymptotic long-range dielectric screening correspond to properties of the exact underlying generalized Kohn-Sham functional, the present results further support that these two properties are physically distinct [157]. The class of global PBE0 $(\alpha)$ hybrid functionals described by the single parameter $\alpha$ is thus clearly insufficient to describe both properties at the same time and thus motivates us to consider more elaborate hybrid functionals.

Next we examine CAM functionals that satisfy Koopmans' condition. These functionals include three parameters $\alpha_{\mathrm{s}}, \alpha_{\ell}$, and $\mu$. Since Koopmans' condition is not sufficient to fix all three parameters, we proceed in the following way. First, we narrow down the parameter space by setting $\alpha_{\ell}$ equal to $1 / \varepsilon_{\infty}^{\text {expt }}$. For the high-frequency dielectric constant $\varepsilon_{\infty}^{\text {expt }}$, we consider 1.77 [81,83] and 1.72 [152] in the cases of liquid water and hexagonal ice, respectively. We remark that taking the experimental value for the dielectric constant introduces an empirical parameter in the scheme. However, this could in principle be eliminated by employing a self-consistent DDH cycle. To avoid an exceedingly complex workflow, we thus set in this work the experimental value from the beginning, thereby ensuring the physically correct screening in the long range. Second, we treat the inverse range-separation length $\mu$ as a free parameter that we vary systematically. Third, we fix $\alpha_{\mathrm{s}}$ through the enforcement of Koopmans' condition. The determined parameter is denoted by $\alpha_{\mathrm{s}, \mathrm{K}}$ and depends parametrically on $\mu$. We note that the resulting functional $\operatorname{CAM}\left(\alpha_{\mathrm{s}, \mathrm{K}}, 1 / \varepsilon_{\infty}^{\mathrm{expt}}, \mu\right)$ incorporates two exact physical constraints, namely, the correct long-range screening as well as Koopmans' condition [157]. The free parameter $\mu$ allows one to mediate between two extreme regimes. Indeed, for $\mu \rightarrow 0$, the present CAM functional reverts to the global hybrid functional $\operatorname{PBE} 0\left(\alpha_{\mathrm{K}}\right)$, which is entirely determined by Koopmans' condition. Instead, for $\mu \rightarrow \infty$, the functional converges asymptotically to the functional $\operatorname{PBE} 0\left(1 / \varepsilon_{\infty}^{\text {expt }}\right)$, which is entirely determined by the dielectric response. In the latter limit, the piecewise linearity can no longer be satisfied.
The continuous variation of $\mu$ allows one to study the bandgap estimate in between these two extreme cases.

For the enforcement of Koopmans' condition to the functional $\operatorname{CAM}\left(\alpha_{\mathrm{s}, \mathrm{K}}, 1 / \varepsilon_{\infty}^{\text {expt }}, \mu\right)$, we adopt the following procedure. We carry out an extended study on hexagonal ice and assume that an analogous analysis would also hold for liquid water insofar as both phases of water exhibit very similar electronic structures. We solely consider the five localized states with the smallest degrees of hybridization, namely, the radical $\mathrm{OH}$, the radical $\mathrm{NH}_{2}$, the fluorine interstitial $\mathrm{F}_{\mathrm{i}}$, the fluorine substitutional $\mathrm{F}_{\mathrm{H}_{2} \mathrm{O}}$, and the chlorine substitutional $\mathrm{Cl}_{\mathrm{H}_{2} \mathrm{O}}$. We verify that the degree of hybridization of these defects remains small when considering the range-separated CAM functional. Thereby, we ensure a reliable enforcement of Koopmans' condition and a consistent comparison with the $\operatorname{PBE} 0\left(\alpha_{\mathrm{K}}\right)$ functional. We evaluate the deviations $\Delta_{\mathrm{K}}^{i}$ for these five localized states and compute the rms deviation $\Delta_{\mathrm{K}}^{\mathrm{rms}}$ using Eq. (5). The minimum of $\Delta_{\mathrm{K}}^{\mathrm{rms}}$ defines $\alpha_{\mathrm{s}, \mathrm{K}}$ for each specific value of the range-separation parameter $\mu$. It is noteworthy that the adopted construction scheme requires numerous supercell calculations at the hybrid functional level. To reduce the computational burden, we first compute $\Delta_{\mathrm{K}}^{\mathrm{rms}}$ using the Bernal-Fowler unit cell with 12 water molecules (cf. Sec. IV A). We use a grid in the two-dimensional space $\left(\alpha_{\mathrm{s}, \mathrm{K}}, \mu\right)$ including six values of $\alpha_{\mathrm{s}, \mathrm{K}}$ and six values of $\mu$. Subsequently, we refine these results using a supercell with 96 water molecules. We generally observe small variations of $\Delta_{\mathrm{K}}^{\mathrm{rms}}$ as compared to the smaller supercell. Hence, the values of the grid obtained with the smaller supercell are adjusted using a linear interpolation of the differences achieved for a $2 \times 2$ subset of relevant points in the space $\left(\alpha_{\mathrm{s}}, \mu\right)$.

Based on the procedure outlined above, we determine $\alpha_{\mathrm{s}, \mathrm{K}}$ as a function of $\mu$. The obtained curve $\left(\alpha_{\mathrm{s}, \mathrm{K}}, \mu\right)$ is referred to as Koopmans' curve and is shown in Fig. 7(a). We observe that $\alpha_{\mathrm{s}, \mathrm{K}}$ decreases continuously with increasing $\mu$. For $\mu=$ 0 , the range-separated CAM functional reverts to the global hybrid functional PBE0 and we thus consistently have $\alpha_{\mathrm{s}, \mathrm{K}}=$ $\alpha_{\mathrm{K}}$. The upper limit for $\mu$ is reached at $\sim 0.75 \mathrm{bohr}^{-1}$, when $\alpha_{\mathrm{s}, \mathrm{K}}$ vanishes. For larger values of $\mu, \Delta_{\mathrm{K}}^{\mathrm{rms}}$ shows no minimum and it is therefore no longer possible to enforce Koopmans' condition.

Next we monitor the band gap along Koopmans' curve highlighted in Fig. 7(a) to identify the range of values consistent with the hybrid functionals $\operatorname{CAM}\left(\alpha_{\mathrm{s}, \mathrm{K}}, 1 / \varepsilon_{\infty}^{\mathrm{expt}}, \mu\right)$. The extracted band gaps are given as a function of $\mu$ in Fig. 7(b). We observe an almost linear increase of the band gap with $\mu$. For vanishing $\mu$, we recover the band gap of $8.9 \mathrm{eV}$ pertaining to the $\operatorname{PBE} 0\left(\alpha_{\mathrm{K}}\right)$ functional. For the upper limit of $\mu$, we achieve a band gap of $9.4 \mathrm{eV}$. This allows us to restrain the range of values achieved with the CAM functionals to $9.15 \pm 0.25 \mathrm{eV}$. We remark that these values are obtained for functionals that reproduce both Koopmans' condition and the asymptotic behavior of the Coulomb interaction. To restrain the value of the band gap even further, it is necessary to invoke additional physical constraints. Similarly to the discussion for selecting the optimal DDH functional, we could use the deviation of the calculated dielectric constant from the experimental value to identify the optimal functional along the Koopmans' curve. However, the calculated dielectric constant is found to vary little among the functionals defined in this 

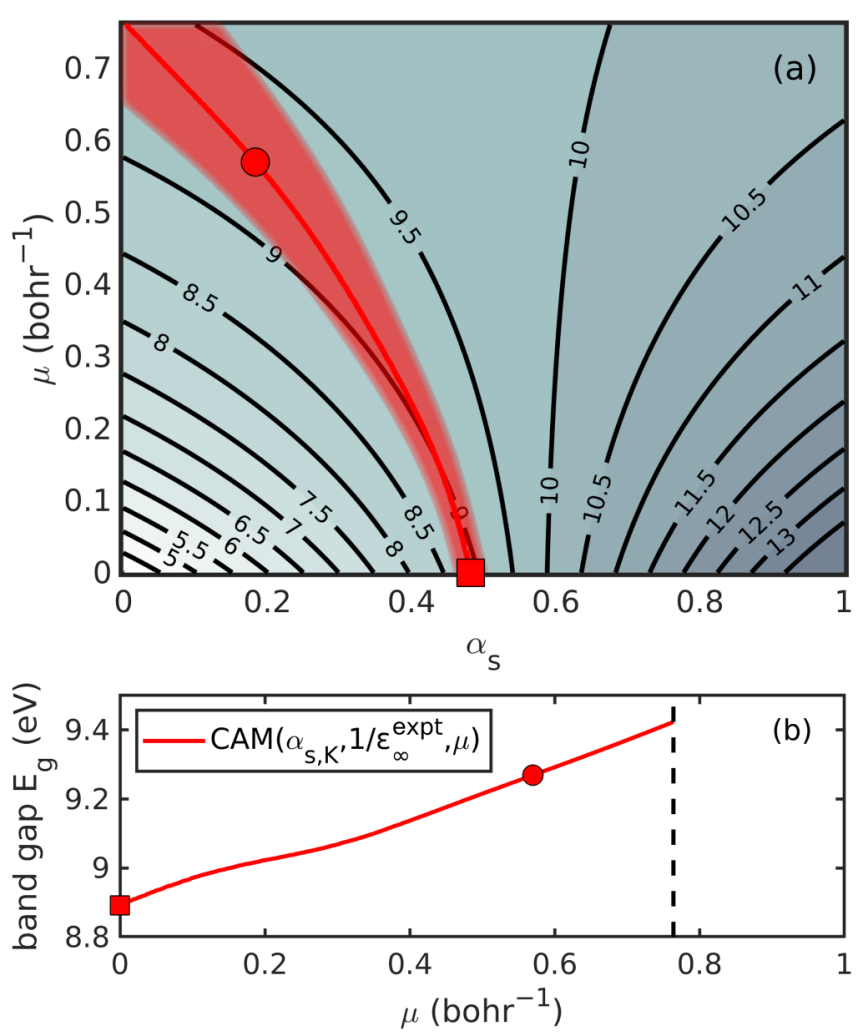

FIG. 7. Band gaps (in eV) of hexagonal ice Ih as obtained with the CAM functional. (a) Isocontour plot for the band gap as obtained with the hybrid functional $\operatorname{CAM}\left(\alpha_{\mathrm{s}}, 1 / \varepsilon_{\infty}^{\exp }, \mu\right)$. The shortrange fraction of Fock exchange $\alpha_{\mathrm{s}}$ and the screening parameter $\mu$ are varied systematically. The red line indicates Koopmans' curve $\left(\alpha_{\mathrm{s}, \mathrm{K}}, \mu\right)$ as obtained through the minimization of $\Delta_{\mathrm{K}}^{\mathrm{rms}}$. The shaded region indicates deviations of less than $0.1 \mathrm{eV}$ from the minimum of $\Delta_{\mathrm{K}}^{\mathrm{rms}}$. (b) Band gaps as obtained with the hybrid functionals $\operatorname{CAM}\left(\alpha_{\mathrm{s}, \mathrm{K}}, 1 / \varepsilon_{\infty}^{\text {expt }}, \mu\right)$ along Koopmans' curve. The vertical dashed line limits the range in which Koopmans' condition can be fulfilled. Data points corresponding to the functionals $\operatorname{PBE} 0\left(\alpha_{\mathrm{K}}\right)$ and $\operatorname{CAM}\left(\alpha_{\mathrm{s}, \mathrm{K}}, 1 / \varepsilon_{\infty}^{\text {expt }}, \mu_{\mathrm{TF}}\right)$ are indicated with squares and circles, respectively. The reported band gaps include the corrections resulting from the ZPR (cf. Sec. II).

way $(1.65 \pm 0.01)$ and thus cannot be used for this purpose. Another physically motivated way to identify the optimal functional consists in realizing that the variation along the Koopmans' curve is determined by the scale of spatial variation in the screening. It is therefore reasonable to adopt the value of $\mu=\mu_{\mathrm{TF}}$ (cf. Sec. III B). For hexagonal ice, $\mu_{\mathrm{TF}}=$ $0.57 \mathrm{bohr}^{-1}$. An explicit fit of the spatially dependent dielectric constant would give $\mu=0.52$ bohr $^{-1}$ [66], corresponding to a band-gap difference of only $0.04 \mathrm{eV}$. This leads us to favor the functional $\operatorname{CAM}\left(\alpha_{\mathrm{s}, \mathrm{K}}, 1 / \varepsilon_{\infty}^{\text {expt }}, \mu_{\mathrm{TF}}\right)$, which gives a band gap of $9.3 \mathrm{eV}$ for hexagonal ice.

In light of the present considerations, the functional $\operatorname{CAM}\left(\alpha_{\mathrm{s}, \mathrm{K}}, 1 / \varepsilon_{\infty}^{\text {expt }}, \mu_{\mathrm{TF}}\right)$ satisfies Koopmans' condition, reproduces the long-range asymptotic potential, and includes the correct length scale for the variation in the screening. This form of hybrid functional should be preferred over the functional $\operatorname{PBE} 0(\alpha)$, which does not possess sufficient free parameters to concomitantly account for all these properties.
Hence, we adopt this form of CAM functional to predict not only the band gap of hexagonal ice but also that of liquid water. In the case of water, we thus adopt $\mu=\mu_{\mathrm{TF}}$ and determine $\alpha_{\mathrm{s}, \mathrm{K}}$ by imposing Koopmans' condition on the same set of five defects employed for hexagonal ice. The consideration of five water snapshots is again sufficient to converge the mean value of $\alpha_{\mathrm{s}, \mathrm{K}}$. The calculated band gaps are given in Table VIII for both phases of water. We observe that the band gaps resulting from the functional $\operatorname{CAM}\left(\alpha_{\mathrm{s}, \mathrm{K}}, 1 / \varepsilon_{\infty}^{\mathrm{expt}}, \mu_{\mathrm{TF}}\right)$ increase by $\sim 0.4-0.5 \mathrm{eV}$ with respect to those obtained with the functional $\operatorname{PBE} 0\left(\alpha_{\mathrm{K}}\right)$. This result is consistent with the trend observed in Fig. 7(b). The agreement with experiment is very good as the band gaps obtained with this CAM functional fall within the range of the experimental reference values.

Before closing this section, it is noteworthy to compare the extension to CAM functionals with results for other materials previously reported in the literature [75,80]. So far, Koopmans' curve has generally been found to remain close to a band-gap isoline, indicating that the range-separated functional does not improve the description achieved with the simpler global hybrid functional. However, in the case of $\mathrm{NaF}$, the band gap obtained with $\operatorname{PBE} 0\left(1 / \varepsilon_{\infty}\right)$ differed from that obtained with $\operatorname{PBE} 0\left(\alpha_{\mathrm{K}}\right)$ by about $0.5 \mathrm{eV}$ and some deviation from the isoline appeared [75]. Similarly, we here find for the two phases of water deviations from the isoline as large as $0.5 \mathrm{eV}$. This suggests that such deviations primarily occur in materials with large band gaps and low dielectric constants. More generally, from the analysis presented above [cf. Fig. 7(b)], such a behavior arises when the band gap achieved with the $\operatorname{PBE} 0\left(\alpha_{\mathrm{K}}\right)$ substantially differs from that obtained with $\operatorname{PBE} 0\left(1 / \varepsilon_{\infty}\right)$.

\section{CONCLUSION}

In this work, we calculated the fundamental band gap of liquid water and hexagonal ice through advanced electronic-structure methods. We investigated specifically the performance of both state-of-the-art $G W$ calculations and nonempirical hybrid functionals. The free parameters of the hybrid functionals were determined either through the dielectric response of the material or the enforcement of Koopmans' condition to localized states. We applied both construction schemes to two classes of hybrid functionals, namely, the global PBE0 and the range-separated CAM functionals. The comparison of such a variety of advanced electronic-structure methods within a consistent computational setup corresponds to one of the main strengths of our work and provides valuable insight into the issue concerning the fundamental band gaps of liquid water and hexagonal ice. The results obtained in this work are summarized in Fig. 8. In addition, we report in Table IX a summary of the parameters of all hybrid functionals retained in this work.

Preliminary to the theoretical investigations, we critically reviewed various experimental studies in the literature. We discussed different estimates for the ionization potential and the electron affinity for both considered water phases. Based on the most reliable results, we inferred experimental values of $9.0 \pm 0.2$ and $9.4 \pm 0.3 \mathrm{eV}$ for the fundamental band gaps of liquid water and hexagonal ice, respectively. These values 


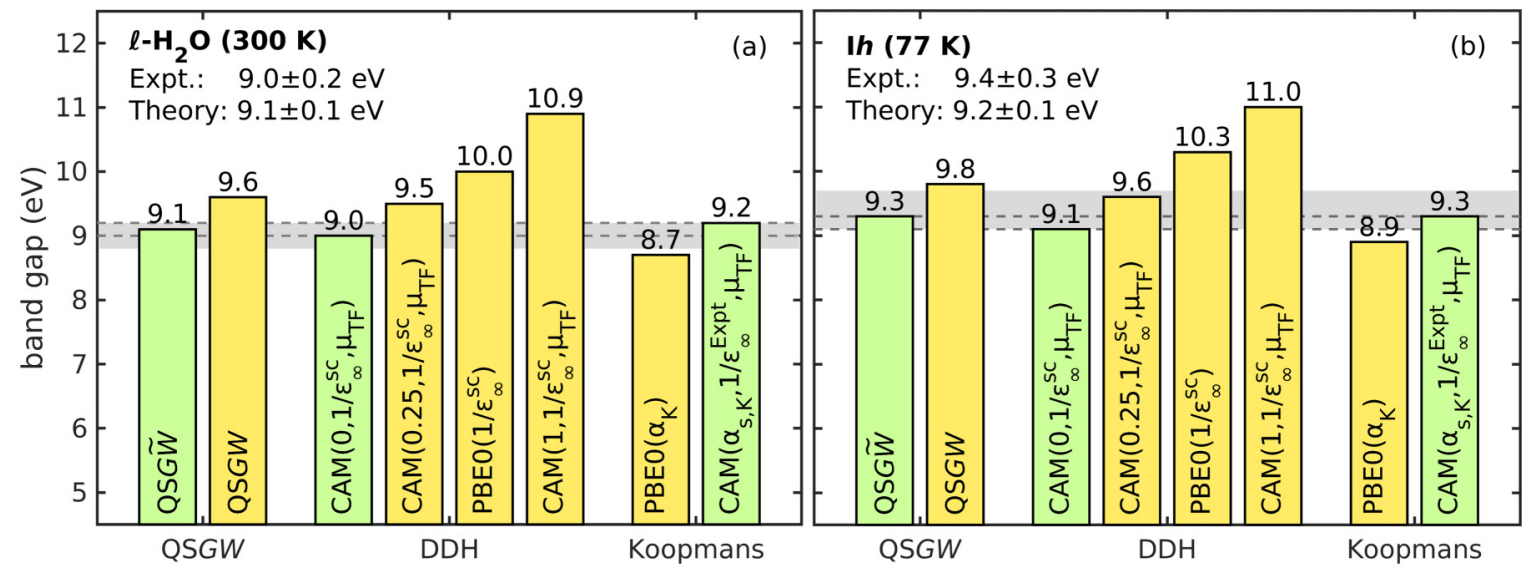

FIG. 8. Fundamental band gaps (in eV) of (a) liquid water $\ell-\mathrm{H}_{2} \mathrm{O}$ and (b) hexagonal ice $\mathrm{Ih}$. Vertical bars indicate the band gaps as calculated with the various advanced electronic-structure methods. The reported band gaps include the corrections associated with NQE and ZPR (cf. Sec. II). The most reliable band-gap calculations are shown in green. Based on these calculations, we infer the range of best theoretical estimates (between dashed lines). The shaded areas indicate the ranges of experimental reference values inferred in Sec. II.

served as robust references for the electronic-structure methods examined in this work.

Then we investigated band gaps as obtained with different state-of-the-art $G W$ calculations. We showed that the QSGW method yields band gaps that overestimate the experimental references. The incorporation of vertex corrections in the screening $(\tilde{W})$ is then instrumental to overcoming the missing electron-hole interaction in $W$. With the resulting QS $G \tilde{W}$ scheme, we found band gaps of 9.1 and $9.3 \mathrm{eV}$ for liquid water and hexagonal ice, respectively. In comparison with the experimental references, these results are in good agreement for both phases of water. Herewith, we confirmed QSGE being one of the most accurate schemes for band-gap predictions $[37,38]$.

Subsequently, we studied dielectric-dependent hybrid functionals for the band gaps of liquid water and hexagonal ice. The band gaps obtained with the global DDH functional overestimate the upper limit of the reference interval by $0.6-$ $0.8 \mathrm{eV}$. Such a poor performance is in line with a previous result for ice [63], but worse than the typical accuracy of this approach for other materials $[63,69]$. Then we studied DDH functionals based on the CAM functional. We consid-

TABLE IX. Parameters of all the hybrid functionals retained in this work describing liquid water $\ell-\mathrm{H}_{2} \mathrm{O}$ and hexagonal ice Ih. The fraction of short-range Fock exchange $\alpha_{\mathrm{s}}$, the fraction of long-range Fock exchange $\alpha_{\ell}$, and the range-separation parameter $\mu$ pertaining to the functional $\operatorname{CAM}\left(\alpha_{\mathrm{s}}, \alpha_{\ell}, \mu\right)$ are given. When $\alpha_{\mathrm{s}}=\alpha_{\ell}=\alpha$, one recovers the functional $\operatorname{PBE} 0(\alpha)$.

\begin{tabular}{|c|c|c|c|c|c|c|}
\hline \multirow[b]{2}{*}{ Functional } & \multicolumn{3}{|c|}{$\ell-\mathrm{H}_{2} \mathrm{O}$} & \multicolumn{3}{|c|}{ Ih } \\
\hline & $\alpha_{\mathrm{s}}$ & $\alpha_{\ell}$ & $\mu$ & $\alpha_{\mathrm{s}}$ & $\alpha_{\ell}$ & $\mu$ \\
\hline $\operatorname{PBE} 0\left(1 / \varepsilon_{\infty}^{\mathrm{sc}}\right)$ & 0.59 & 0.59 & & 0.61 & 0.61 & \\
\hline $\operatorname{CAM}\left(1,1 / \varepsilon_{\infty}^{\mathrm{sc}}, \mu_{\mathrm{TF}}\right)$ & 1 & 0.60 & 0.58 & 1 & 0.62 & 0.57 \\
\hline $\operatorname{CAM}\left(0.25,1 / \varepsilon_{\infty}^{\mathrm{sc}}, \mu_{\mathrm{TF}}\right)$ & 0.25 & 0.58 & 0.58 & 0.25 & 0.60 & 0.57 \\
\hline $\operatorname{CAM}\left(0,1 / \varepsilon_{\infty}^{\mathrm{sc}}, \mu_{\mathrm{TF}}\right)$ & 0 & 0.57 & 0.58 & 0 & 0.59 & 0.57 \\
\hline $\operatorname{PBE} 0\left(\alpha_{\mathrm{K}}\right)$ & 0.47 & 0.47 & & 0.48 & 0.48 & \\
\hline $\operatorname{CAM}\left(\alpha_{\mathrm{s}, \mathrm{K}}, 1 / \varepsilon_{\infty}^{\text {expt }}, \mu_{\mathrm{TF}}\right)$ & 0.18 & 0.56 & 0.58 & 0.18 & 0.58 & 0.57 \\
\hline
\end{tabular}

ered three range-separated DDH functionals differing in the short-range fraction of Fock exchange. Based on the comparison with experimental references for the dielectric constant, we identified the functional $\operatorname{CAM}\left(0,1 / \varepsilon_{\infty}^{\mathrm{sc}}, \mu_{\mathrm{TF}}\right)$ as the most reliable DDH functional. This functional incorporates pure semilocal exchange in the short range, thereby extending the scope of long-range corrected hybrid functionals [121-125]. This scheme yields band gaps of 9.0 and $9.1 \mathrm{eV}$ for liquid water and hexagonal ice, respectively.

Finally, we investigated hybrid functionals satisfying Koopmans' condition. We found that the enforcement of this physical constraint also gives accurate band gaps. The minimization of the degree of hybridization between localized defects and delocalized band-edge states is thereby key to ensuring proper band-gap predictions. First, we constructed the global functional $\operatorname{PBE} 0\left(\alpha_{\mathrm{K}}\right)$ and achieved band gaps for liquid water and hexagonal ice of 8.7 and $8.9 \mathrm{eV}$, respectively. These results lie lower than the onset of the range of experimental references by only $0.1-0.2 \mathrm{eV}$. This is in contrast to the global DDH functional, which is unable to attain such an accuracy. Then we constructed range-separated hybrid functionals that satisfy Koopmans' condition. In particular, we investigated the functional $\operatorname{CAM}\left(\alpha_{\mathrm{s}, \mathrm{K}}, 1 / \varepsilon_{\infty}^{\text {expt }}, \mu\right)$, which additionally enforces the correct long-range screening [157]. The obtained band-gap estimate exhibits a parametric dependence on the range-separation parameter $\mu$ encompassing the result of the global hybrid functional $\operatorname{PBE} 0\left(\alpha_{\mathrm{K}}\right)$ as a lower bound. We overcame the $\mu$ dependence by invoking the scale of spatial variation of the screening, i.e., $\mu=\mu_{\mathrm{TF}}$. This nonempirical and physically motivated setting leads to the functional $\operatorname{CAM}\left(\alpha_{\mathrm{s}, \mathrm{K}}, 1 / \varepsilon_{\infty}^{\text {expt }}, \mu_{\mathrm{TF}}\right)$. We employed this functional for the band-gap estimates of liquid water and hexagonal ice and found 9.2 and $9.3 \mathrm{eV}$, respectively.

Overall, we showed that the three advanced electronicstructure methods considered in this work provide consistent results for the band gaps of liquid water and hexagonal ice. Indeed, the band gaps of the most reliable schemes, namely, $\mathrm{QS} G \tilde{W}, \operatorname{CAM}\left(0,1 / \varepsilon_{\infty}^{\mathrm{sc}}, \mu_{\mathrm{TF}}\right)$, and $\operatorname{CAM}\left(\alpha_{\mathrm{s}, \mathrm{K}}, 1 / \varepsilon_{\infty}^{\mathrm{expt}}, \mu_{\mathrm{TF}}\right)$, differ by at most $0.2 \mathrm{eV}$, which corresponds to only $\sim 2 \%$ of the band gaps involved. We remark that this agreement 
originates from a consistent computational setup and from the critical consideration of each method. In this way, our analysis allows us to resolve the discord arising from previous studies. Based on the present results, we inferred best theoretical estimates of $9.1 \pm 0.1$ and $9.2 \pm 0.1 \mathrm{eV}$ for liquid water and hexagonal ice, respectively. The comparison of these theoretical ranges with the experimental ones obtained in Sec. II indicates a remarkable agreement. The present comparison provides strong evidence for the reliability of both experimental and theoretical estimates and reduces the residual uncertainty on the fundamental band gaps of liquid water and hexagonal ice.

Additionally to the accuracy in the band-gap predictions, it is of interest to compare also the computational costs and the robustness of the examined electronic-structure methods. Quasiparticle self-consistent $G W$ calculations require extensive computational resources and remain therefore limited to relatively small unit cells. Nevertheless, the reliability of the QSGW scheme has been verified not only for water and ice but also for numerous other materials. Dielectric-dependent hybrid functionals have the potential to replace such highlevel $G W$ methods due to their efficient implementation in many electronic-structure codes and their fast convergence within only a few iterations. However, the present results indicate that the band gaps obtained with DDH functionals can span a large interval of values, leading to considerable uncertainty. In this work, we overcame this problem by taking into consideration the description of the dielectric constant $\varepsilon_{\infty}$, but the validity of this way of proceeding for a larger variety of materials remains to be ascertained. Hybrid functionals satisfying Koopmans' condition also serve as a viable alternative to QSGW . This approach yields robust band-gap estimates irrespective of the considered class of hybrid functionals. However, the application of this method generally requires defect calculations at the hybrid functional level with relatively large supercells, thereby limiting its potential.

In view of employing the present electronic-structure methods to applications involving liquid water and hexagonal ice, it is clear that the hybrid functional approaches offer significant advantages in terms of computational cost and the availability of a total-energy scheme. The present work shows that the compromise on accuracy is limited as the hybrid functional approaches achieve band gaps comparable to those obtained with the most elaborate $G W$ scheme.

\section{ACKNOWLEDGMENTS}

We thank Wei Chen, Giacomo Miceli, Francesco Ambrosio, and Alexey Tal for fruitful discussions. Support from the Swiss National Science Foundation is acknowledged under Grant No. 200020-172524. The calculations have been performed at the Swiss National Supercomputing Center (CSCS) (grant under Project No. s879) and at SCITAS EPFL.
[1] D. Grand, A. Bernas, and E. Amouyal, Photoionization of aqueous indole: Conduction band edge and energy gap in liquid water, Chem. Phys. 44, 73 (1979).

[2] A. Bernas, D. Grand, and E. Amouyal, Photoionization of solutes and conduction band edge of solvents. Indole in water and alcohols, J. Phys. Chem. 84, 1259 (1980).

[3] I. Watanabe, J. B. Flanagan, and P. Delahay, Vacuum ultraviolet photoelectron emission spectroscopy of water and aqueous solutions, J. Chem. Phys. 73, 2057 (1980).

[4] P. Delahay and K. Von Burg, Photoelectron emission spectroscopy of liquid water, Chem. Phys. Lett. 83, 250 (1981).

[5] A. Bernas, C. Ferradini, and J.-P. Jay-Gerin, On the electronic structure of liquid water: Facts and reflections, Chem. Phys. 222, 151 (1997).

[6] B. Winter, R. Weber, W. Widdra, M. Dittmar, M. Faubel, and I. V. Hertel, Full valence band photoemission from liquid water using EUV synchrotron radiation, J. Phys. Chem. A 108, 2625 (2004).

[7] T. A. Pham, C. Zhang, E. Schwegler, and G. Galli, Probing the electronic structure of liquid water with many-body perturbation theory, Phys. Rev. B 89, 060202(R) (2014).

[8] W. Chen, F. Ambrosio, G. Miceli, and A. Pasquarello, Ab initio Electronic Structure of Liquid Water, Phys. Rev. Lett. 117, 186401 (2016).

[9] A. P. Gaiduk, T. A. Pham, M. Govoni, F. Paesani, and G. Galli, Electron affinity of liquid water, Nat. Commun. 9, 247 (2018).

[10] F. Ambrosio, Z. Guo, and A. Pasquarello, Absolute energy levels of liquid water, J. Phys. Chem. Lett. 9, 3212 (2018).

[11] F. Ambrosio, G. Miceli, and A. Pasquarello, Redox levels in aqueous solution: Effect of van der Waals interactions and hybrid functionals, J. Chem. Phys. 143, 244508 (2015).
[12] J. Wiktor, F. Bruneval, and A. Pasquarello, Partial molar volumes of aqua ions from first principles, J. Chem. Theory Comput. 13, 3427 (2017).

[13] T. A. Pham, M. Govoni, R. Seidel, S. E. Bradforth, E. Schwegler, and G. Galli, Electronic structure of aqueous solutions: Bridging the gap between theory and experiments, Sci. Adv. 3, e1603210 (2017).

[14] F. Ambrosio, G. Miceli, and A. Pasquarello, Electronic levels of excess electrons in liquid water, J. Phys. Chem. Lett. 8, 2055 (2017).

[15] M. Pizzochero, F. Ambrosio, and A. Pasquarello, Picture of the wet electron: A localized transient state in liquid water, Chem. Sci. 10, 7442 (2019).

[16] N. Kharche, J. T. Muckerman, and M. S. Hybertsen, FirstPrinciples Approach to Calculating Energy Level Alignment at Aqueous Semiconductor Interfaces, Phys. Rev. Lett. 113, 176802 (2014).

[17] M. Govoni and G. Galli, Large scale GW calculations, J. Chem. Theory Comput. 11, 2680 (2015).

[18] Z. Guo, F. Ambrosio, W. Chen, P. Gono, and A. Pasquarello, Alignment of redox levels at semiconductor-water interfaces, Chem. Mater. 30, 94 (2018).

[19] H. Zheng, M. Govoni, and G. Galli, Dielectric-dependent hybrid functionals for heterogeneous materials, Phys. Rev. Mater. 3, 073803 (2019).

[20] A. Bouzid, P. Gono, and A. Pasquarello, Reaction pathway of oxygen evolution on $\operatorname{Pt}(111)$ revealed through constant Fermi level molecular dynamics, J. Catal. 375, 135 (2019).

[21] P. Gono, F. Ambrosio, and A. Pasquarello, Effect of the solvent on the oxygen evolution reaction at the $\mathrm{TiO}_{2}$-water interface, J. Phys. Chem. C 123, 18467 (2019). 
[22] J. Klánová, P. Klán, J. Nosek, and I. Holoubek, Environmental ice photochemistry: Monochlorophenols, Environ. Sci. Technol. 37, 1568 (2003).

[23] C. S. Boxe, A. J. Colussi, M. R. Hoffmann, I. M. Perez, J. G. Murphy, and R. C. Cohen, Kinetics of $\mathrm{NO}$ and $\mathrm{NO}_{2}$ evolution from illuminated frozen nitrate solutions, J. Phys. Chem. A 110, 3578 (2006).

[24] N. Takenaka and H. Bandow, Chemical kinetics of reactions in the unfrozen solution of ice, J. Phys. Chem. A 111, 8780 (2007).

[25] A. M. Grannas, A. E. Jones, J. Dibb, M. Ammann, C. Anastasio, H. J. Beine, M. Bergin, J. Bottenheim, C. S. Boxe, G. Carver, and G. Chen, An overview of snow photochemistry: Evidence, mechanisms and impacts, Atmos. Chem. Phys. 7, 4329 (2007).

[26] P. Llombart, E. G. Noya, and L. G. MacDowell, Surface phase transitions and crystal habits of ice in the atmosphere, Sci. Adv. 6, eaay9322 (2020).

[27] A. Liebscher, Aqueous fluids at elevated pressure and temperature, Geofluids 10, 3 (2010).

[28] P. Jenniskens and D. F. Blake, Structural transitions in amorphous water ice and astrophysical implications, Science 265, 753 (1994).

[29] L. Amiaud, A. Momeni, F. Dulieu, J. H. Fillion, E. Matar, and J.-L. Lemaire, Measurement of the Adsorption Energy Difference between Ortho- and Para- $\mathrm{D}_{2}$ on an Amorphous Ice Surface, Phys. Rev. Lett. 100, 056101 (2008).

[30] C. S. Cucinotta, I. Rungger, and S. Sanvito, First principles study of electron tunneling through ice, J. Phys. Chem. C 116, 22129 (2012).

[31] K. Thürmer and S. Nie, Formation of hexagonal and cubic ice during low-temperature growth, Proc. Natl. Acad. Sci. USA 110, 11757 (2013)

[32] G. C. Sosso, T. F. Whale, M. A. Holden, P. Pedevilla, B. J. Murray, and A. Michaelides, Unravelling the origins of ice nucleation on organic crystals, Chem. Sci. 9, 8077 (2018).

[33] N. Gerrard, C. Gattinoni, F. McBride, A. Michaelides, and A. Hodgson, Strain relief during ice growth on a hexagonal template, J. Am. Chem. Soc. 141, 8599 (2019).

[34] E. A. Engel, B. Monserrat, and R. J. Needs, Vibrational renormalisation of the electronic band gap in hexagonal and cubic ice, J. Chem. Phys. 143, 244708 (2015).

[35] L. Hedin, New method for calculating the one-particle Green's function with application to the electron-gas problem, Phys. Rev. 139, A796 (1965).

[36] M. van Schilfgaarde, T. Kotani, and S. Faleev, Quasiparticle Self-Consistent GW Theory, Phys. Rev. Lett. 96, 226402 (2006).

[37] M. Shishkin, M. Marsman, and G. Kresse, Accurate Quasiparticle Spectra from Self-Consistent GW Calculations with Vertex Corrections, Phys. Rev. Lett. 99, 246403 (2007).

[38] W. Chen and A. Pasquarello, Accurate band gaps of extended systems via efficient vertex corrections in $G W$, Phys. Rev. B 92, 041115(R) (2015).

[39] V. Garbuio, M. Cascella, L. Reining, R. Del Sole, and O. Pulci, $A b$ initio Calculation of Optical Spectra of Liquids: Many-Body Effects in the Electronic Excitations of Water, Phys. Rev. Lett. 97, 137402 (2006).
[40] D. Lu, F. Gygi, and G. Galli, Dielectric Properties of Ice and Liquid Water from First-Principles Calculations, Phys. Rev. Lett. 100, 147601 (2008).

[41] V. Ziaei and T. Bredow, Probing ionization potential, electron affinity and self-energy effect on the spectral shape and exciton binding energy of quantum liquid water with self-consistent many-body perturbation theory and the Bethe-Salpeter equation, J. Phys.: Condens. Matter 30, 215502 (2018).

[42] P. H. Hahn, W. G. Schmidt, K. Seino, M. Preuss, F. Bechstedt, and J. Bernholc, Optical Absorption of Water: Coulomb Effects versus Hydrogen Bonding, Phys. Rev. Lett. 94, 037404 (2005)

[43] C. Fang, W.-F. Li, R. S. Koster, J. Klimeš, A. van Blaaderen, and M. A. van Huis, The accurate calculation of the band gap of liquid water by means of $G W$ corrections applied to plane-wave density functional theory molecular dynamics simulations, Phys. Chem. Chem. Phys. 17, 365 (2015).

[44] W. L. Yim, H. Shi, Y. Liang, R. J. Hemley, and J. S. Tse, in Correlations in Condensed Matter under Extreme Conditions, edited by G. G. N. Angilella and A. La Magna (Springer, Cham, 2017), pp. 107-126.

[45] A. D. Becke, A new mixing of Hartree-Fock and local densityfunctional theories, J. Chem. Phys. 98, 1372 (1993).

[46] J. P. Perdew, M. Ernzerhof, and K. Burke, Rationale for mixing exact exchange with density functional approximations, J. Chem. Phys. 105, 9982 (1996).

[47] J. Heyd, G. E. Scuseria, and M. Ernzerhof, Hybrid functionals based on a screened Coulomb potential, J. Chem. Phys. 118, 8207 (2003)

[48] T. Yanai, D. P. Tew, and N. C. Handy, A new hybrid exchangecorrelation functional using the Coulomb-attenuating method (CAM-B3LYP), Chem. Phys. Lett. 393, 51 (2004).

[49] D. Pan, Q. Wan, and G. Galli, The refractive index and electronic gap of water and ice increase with increasing pressure, Nat. Commun. 5, 3919 (2014).

[50] A. P. Gaiduk, F. Gygi, and G. Galli, Density and compressibility of liquid water and ice from first-principles simulations with hybrid functionals, J. Phys. Chem. Lett. 6, 2902 (2015).

[51] F. Ambrosio, G. Miceli, and A. Pasquarello, Structural, dynamical, and electronic properties of liquid water: A hybrid functional study, J. Phys. Chem. B 120, 7456 (2016).

[52] B. Cheng, E. A. Engel, J. Behler, C. Dellago, and M. Ceriotti, $\mathrm{Ab}$ initio thermodynamics of liquid and solid water, Proc. Natl. Acad. Sci. USA 116, 1110 (2019).

[53] M. Levy, J. P. Perdew, and V. Sahni, Exact differential equation for the density and ionization energy of a many-particle system, Phys. Rev. A 30, 2745 (1984).

[54] A. Alkauskas, P. Broqvist, and A. Pasquarello, Defect levels through hybrid density functionals: Insights and applications, Phys. Status Solidi B 248, 775 (2011).

[55] M. A. L. Marques, J. Vidal, M. J. T. Oliveira, L. Reining, and S. Botti, Density-based mixing parameter for hybrid functionals, Phys. Rev. B 83, 035119 (2011).

[56] S. Refaely-Abramson, S. Sharifzadeh, M. Jain, R. Baer, J. B. Neaton, and L. Kronik, Gap renormalization of molecular crystals from density-functional theory, Phys. Rev. B 88, 081204(R) (2013).

[57] J. P. Perdew, R. G. Parr, M. Levy, and J. L. Balduz, Density-Functional Theory for Fractional Particle Number: 
Derivative Discontinuities of the Energy, Phys. Rev. Lett. 49, 1691 (1982).

[58] W. Yang, Y. Zhang, and P. W. Ayers, Degenerate Ground States and a Fractional Number of Electrons in Density and Reduced Density Matrix Functional Theory, Phys. Rev. Lett. 84, 5172 (2000).

[59] T. Shimazaki and Y. Asai, Band structure calculations based on screened Fock exchange method, Chem. Phys. Lett. 466, 91 (2008).

[60] T. Shimazaki and Y. Asai, First principles band structure calculations based on self-consistent screened HartreeFock exchange potential, J. Chem. Phys. 130, 164702 (2009).

[61] T. Shimazaki and Y. Asai, Energy band structure calculations based on screened Hartree-Fock exchange method: Si, AlP, AlAs, GaP, and GaAs, J. Chem. Phys. 132, 224105 (2010).

[62] S. Refaely-Abramson, S. Sharifzadeh, N. Govind, J. Autschbach, J. B. Neaton, R. Baer, and L. Kronik, Quasiparticle Spectra from a Nonempirical Optimally Tuned Range-Separated Hybrid Density Functional, Phys. Rev. Lett. 109, 226405 (2012).

[63] J. H. Skone, M. Govoni, and G. Galli, Self-consistent hybrid functional for condensed systems, Phys. Rev. B 89, 195112 (2014).

[64] M. Gerosa, C. E. Bottani, L. Caramella, G. Onida, C. Di Valentin, and G. Pacchioni, Electronic structure and phase stability of oxide semiconductors: Performance of dielectricdependent hybrid functional DFT, benchmarked against $G W$ band structure calculations and experiments, Phys. Rev. B 91, 155201 (2015).

[65] M. Gerosa, C. Di Valentin, C. E. Bottani, G. Onida, and G. Pacchioni, Communication: Hole localization in Al-doped quartz $\mathrm{SiO}_{2}$ within ab initio hybrid-functional DFT, J. Chem. Phys. 143, 111103 (2015).

[66] J. H. Skone, M. Govoni, and G. Galli, Nonempirical rangeseparated hybrid functionals for solids and molecules, Phys. Rev. B 93, 235106 (2016).

[67] M. Gerosa, C. E. Bottani, C. Di Valentin, G. Onida, and G. Pacchioni, Accuracy of dielectric-dependent hybrid functionals in the prediction of optoelectronic properties of metal oxide semiconductors: A comprehensive comparison with manybody $G W$ and experiments, J. Phys.: Condens. Matter 30, 044003 (2017).

[68] Z.-H. Cui, Y.-C. Wang, M.-Y. Zhang, X. Xu, and H. Jiang, Doubly screened hybrid functional: An accurate firstprinciples approach for both narrow- and wide-gap semiconductors, J. Phys. Chem. Lett. 9, 2338 (2018).

[69] W. Chen, G. Miceli, G.-M. Rignanese, and A. Pasquarello, Nonempirical dielectric-dependent hybrid functional with range separation for semiconductors and insulators, Phys. Rev. Mater. 2, 073803 (2018).

[70] A. P. Gaiduk, J. Gustafson, F. Gygi, and G. Galli, Firstprinciples simulations of liquid water using a dielectricdependent hybrid functional, J. Phys. Chem. Lett. 9, 3068 (2018).

[71] P. Liu, C. Franchini, M. Marsman, and G. Kresse, Assessing model-dielectric-dependent hybrid functionals on the antiferromagnetic transition-metal monoxides $\mathrm{MnO}, \mathrm{FeO}, \mathrm{CoO}$, and NiO, J. Phys.: Condens. Matter 32, 015502 (2019).
[72] B. Sadigh, P. Erhart, and D. Åberg, Variational polaron self-interaction-corrected total-energy functional for charge excitations in insulators, Phys. Rev. B 92, 075202 (2015).

[73] P. Deák, Q. D. Ho, F. Seemann, B. Aradi, M. Lorke, and T. Frauenheim, Choosing the correct hybrid for defect calculations: A case study on intrinsic carrier trapping in $\beta-\mathrm{Ga}_{2} \mathrm{O}_{3}$, Phys. Rev. B 95, 075208 (2017).

[74] S. Kokott, S. V. Levchenko, P. Rinke, and M. Scheffler, Firstprinciples supercell calculations of small polarons with proper account for long-range polarization effects, New J. Phys. 20, 033023 (2018).

[75] G. Miceli, W. Chen, I. Reshetnyak, and A. Pasquarello, Nonempirical hybrid functionals for band gaps and polaronic distortions in solids, Phys. Rev. B 97, 121112(R) (2018).

[76] T. J. Smart, F. Wu, M. Govoni, and Y. Ping, Fundamental principles for calculating charged defect ionization energies in ultrathin two-dimensional materials, Phys. Rev. Mater. 2 , 124002 (2018).

[77] A. R. Elmaslmane, M. B. Watkins, and K. P. McKenna, Firstprinciples modeling of polaron formation in $\mathrm{TiO}_{2}$ polymorphs, J. Chem. Theory Comput. 14, 3740 (2018).

[78] T. Bischoff, I. Reshetnyak, and A. Pasquarello, Adjustable potential probes for band-gap predictions of extended systems through nonempirical hybrid functionals, Phys. Rev. B 99, 201114(R) (2019).

[79] P. Deák, M. Lorke, B. Aradi, and T. Frauenheim, Optimized hybrid functionals for defect calculations in semiconductors, J. Appl. Phys. 126, 130901 (2019).

[80] T. Bischoff, J. Wiktor, W. Chen, and A. Pasquarello, Nonempirical hybrid functionals for band gaps of inorganic metalhalide perovskites, Phys. Rev. Mater. 3, 123802 (2019).

[81] J. M. Heller, R. N. Hamm, R. D. Birkhoff, and L. R. Painter, Collective oscillation in liquid water, J. Chem. Phys. 60, 3483 (1974).

[82] K. Kobayashi, Optical spectra and electronic structure of ice, J. Phys. Chem. 87, 4317 (1983).

[83] H. Hayashi and N. Hiraoka, Accurate measurements of dielectric and optical functions of liquid water and liquid benzene in the VUV region $(1-100 \mathrm{eV})$ using small-angle inelastic $\mathrm{x}$-ray scattering, J. Phys. Chem. B 119, 5609 (2015).

[84] N. L. Nguyen, H. Ma, M. Govoni, F. Gygi, and G. Galli, FiniteField Approach to Solving the Bethe-Salpeter Equation, Phys. Rev. Lett. 122, 237402 (2019).

[85] J. V. Coe, A. D. Earhart, M. H. Cohen, G. J. Hoffman, H. W. Sarkas, and K. H. Bowen, Using cluster studies to approach the electronic structure of bulk water: Reassessing the vacuum level, conduction band edge, and band gap of water, J. Chem. Phys. 107, 6023 (1997).

[86] L. R. Painter, R. N. Hamm, E. T. Arakawa, and R. D. Birkhoff, Electronic Properties of Liquid Water in the Vacuum Ultraviolet, Phys. Rev. Lett. 21, 282 (1968).

[87] J.-Y. Chen and C.-S. Yoo, High density amorphous ice at room temperature, Proc. Natl. Acad. Sci. USA 108, 7685 (2011).

[88] D. Nordlund, M. Odelius, H. Bluhm, H. Ogasawara, L. G. M. Pettersson, and A. Nilsson, Electronic structure effects in liquid water studied by photoelectron spectroscopy and density functional theory, Chem. Phys. Lett. 460, 86 (2008).

[89] T. Shibaguchi, H. Onuki, and R. Onaka, Electronic structures of water and ice, J. Phys. Soc. Jpn. 42, 152 (1977). 
[90] J. Stähler, J.-C. Deinert, D. Wegkamp, S. Hagen, and M. Wolf, Real-time measurement of the vertical binding energy during the birth of a solvated electron, J. Am. Chem. Soc. 137, 3520 (2015).

[91] B. Baron, D. Hoover, and F. Williams, Vacuum ultraviolet photoelectric emission from amorphous ice, J. Chem. Phys. 68, 1997 (1978).

[92] M. J. Campbell, J. Liesegang, J. D. Riley, R. C. G. Leckey, J. G. Jenkin, and R. T. Poole, The electronic structure of the valence bands of solid $\mathrm{NH}_{3}$ and $\mathrm{H}_{2} \mathrm{O}$ studied by ultraviolet photoelectron spectroscopy, J. Electron Spectrosc. Relat. Phenom. 15, 83 (1979).

[93] T. Pache, H.-P. Steinrück, W. Huber, and D. Menzel, The adsorption of $\mathrm{H}_{2} \mathrm{O}$ on clean and oxygen precovered $\mathrm{Ni}(111)$ studied by ARUPS and TPD, Surf. Sci. 224, 195 (1989).

[94] M. Michaud, P. Cloutier, and L. Sanche, Low-energy electronenergy-loss spectroscopy of amorphous ice: Electronic excitations, Phys. Rev. A 44, 5624 (1991).

[95] D. Grand and A. Bernas, On the conduction band edge energy of ice, Chem. Phys. Lett. 97, 119 (1983).

[96] J. V. Coe, G. H. Lee, J. G. Eaton, S. T. Arnold, H. W. Sarkas, K. H. Bowen, C. Ludewigt, H. Haberland, and D. R. Worsnop, Photoelectron spectroscopy of hydrated electron cluster anions, $\left(\mathrm{H}_{2} \mathrm{O}\right)_{n=2-69}^{-}$, J. Chem. Phys. 92, 3980 (1990).

[97] L. Kevan, Energy level structure and mobilities of excess electrons in aqueous and organic glasses, J. Phys. Chem. 76, 3830 (1972).

[98] B. Monserrat, E. A. Engel, and R. J. Needs, Giant electronphonon interactions in molecular crystals and the importance of nonquadratic coupling, Phys. Rev. B 92, 140302(R) (2015).

[99] M. Del Ben, J. Hutter, and J. Vande Vondele, Probing the structural and dynamical properties of liquid water with models including non-local electron correlation, J. Chem. Phys. 143, 054506 (2015).

[100] J. P. Perdew, K. Burke, and M. Ernzerhof, Generalized Gradient Approximation Made Simple, Phys. Rev. Lett. 77, 3865 (1996).

[101] W. Chen, F. Ambrosio, G. Miceli, and A. Pasquarello, Ab initio electronic structure of liquid water: Molecular dynamics snapshots, Materials Cloud Archive 2018.0023/v1 (2018), doi:10.24435/materialscloud:2018.0023/v1.

[102] M. S. Hybertsen and S. G. Louie, Electron correlation in semiconductors and insulators: Band gaps and quasiparticle energies, Phys. Rev. B 34, 5390 (1986).

[103] F. Gygi and A. Baldereschi, Quasiparticle Energies in Semiconductors: Self-Energy Correction to the Local-Density Approximation, Phys. Rev. Lett. 62, 2160 (1989).

[104] W. Chen and A. Pasquarello, Band-edge positions in $G W$ : Effects of starting point and self-consistency, Phys. Rev. B 90, 165133 (2014)

[105] J. Klimes, M. Kaltak, and G. Kresse, Predictive $G W$ calculations using plane waves and pseudopotentials, Phys. Rev. B 90, 075125 (2014).

[106] L. J. Sham and M. Schlüter, Density-Functional Theory of the Energy Gap, Phys. Rev. Lett. 51, 1888 (1983).

[107] F. Bruneval, N. Vast, L. Reining, M. Izquierdo, F. Sirotti, and N. Barrett, Exchange and Correlation Effects in Electronic Excitations of $\mathrm{Cu}_{2} \mathrm{O}$, Phys. Rev. Lett. 97, 267601 (2006).

[108] J. Vidal, S. Botti, P. Olsson, J.-F. Guillemoles, and L. Reining, Strong Interplay between Structure and Electronic Properties in $\mathrm{CuIn}(\mathrm{S}, \mathrm{Se})_{2}$ : A First-Principles Study, Phys. Rev. Lett. 104, 056401 (2010).

[109] S. V. Faleev, M. van Schilfgaarde, and T. Kotani, All-Electron Self-Consistent $G W$ Approximation: Application to $\mathrm{Si}, \mathrm{MnO}$, and NiO, Phys. Rev. Lett. 93, 126406 (2004).

[110] F. Bruneval, N. Vast, and L. Reining, Effect of self-consistency on quasiparticles in solids, Phys. Rev. B 74, 045102 (2006).

[111] T. Kotani, M. van Schilfgaarde, and S. V. Faleev, Quasiparticle self-consistent $G W$ method: A basis for the independentparticle approximation, Phys. Rev. B 76, 165106 (2007).

[112] S. Botti, D. Kammerlander, and M. A. L. Marques, Band structures of $\mathrm{Cu}_{2} \mathrm{ZnSnS}_{4}$ and $\mathrm{Cu}_{2} \mathrm{ZnSnSe}_{4}$ from many-body methods, Appl. Phys. Lett. 98, 241915 (2011).

[113] B. Holm and U. von Barth, Fully self-consistent GW selfenergy of the electron gas, Phys. Rev. B 57, 2108 (1998).

[114] S. Sharma, J. K. Dewhurst, A. Sanna, and E. K. U. Gross, Bootstrap Approximation for the Exchange-Correlation Kernel of Time-Dependent Density-Functional Theory, Phys. Rev. Lett. 107, 186401 (2011).

[115] T. Bischoff, I. Reshetnyak, and A. Pasquarello, Band alignment at the $\mathrm{CaF}_{2} / \mathrm{Si}(111)$ interface through advanced electronic structure calculations, Phys. Rev. B 101, 235302 (2020).

[116] J. Wiktor, I. Reshetnyak, F. Ambrosio, and A. Pasquarello, Comprehensive modeling of the band gap and absorption spectrum of $\mathrm{BiVO}_{4}$, Phys. Rev. Mater. 1, 022401(R) (2017).

[117] C. Bhandari, M. van Schilfgaarde, T. Kotani, and W. R. L. Lambrecht, All-electron quasiparticle self-consistent $G W$ band structures for $\mathrm{SrTiO}_{3}$ including lattice polarization corrections in different phases, Phys. Rev. Mater. 2, 013807 (2018).

[118] J. Wiktor, U. Rothlisberger, and A. Pasquarello, Predictive determination of band gaps of inorganic halide perovskites, J. Phys. Chem. Lett. 8, 5507 (2017).

[119] A. Seidl, A. Görling, P. Vogl, J. A. Majewski, and M. Levy, Generalized Kohn-Sham schemes and the band-gap problem, Phys. Rev. B 53, 3764 (1996).

[120] J. Heyd, G. E. Scuseria, and M. Ernzerhof, Erratum: "Hybrid functionals based on a screened coulomb potential" [J. Chem. Phys. 118, 8207 (2003)], J. Chem. Phys. 124, 219906 (2006).

[121] T. Leininger, H. Stoll, H.-J. Werner, and A. Savin, Combining long-range configuration interaction with short-range density functionals, Chem. Phys. Lett. 275, 151 (1997).

[122] H. Iikura, T. Tsuneda, T. Yanai, and K. Hirao, A long-range correction scheme for generalized-gradient-approximation exchange functionals, J. Chem. Phys. 115, 3540 (2001).

[123] R. Baer and D. Neuhauser, Density Functional Theory with Correct Long-Range Asymptotic Behavior, Phys. Rev. Lett. 94, 043002 (2005).

[124] I. C. Gerber, J. G. Ángyán, M. Marsman, and G. Kresse, Range separated hybrid density functional with long-range HartreeFock exchange applied to solids, J. Chem. Phys. 127, 054101 (2007).

[125] J.-D. Chai and M. Head-Gordon, Systematic optimization of long-range corrected hybrid density functionals, J. Chem. Phys. 128, 084106 (2008).

[126] P. Mori-Sánchez, A. J. Cohen, and W. Yang, Many-electron self-interaction error in approximate density functionals, J. Chem. Phys. 125, 201102 (2006).

[127] P. Mori-Sánchez, A. J. Cohen, and W. Yang, Localization and Delocalization Errors in Density Functional Theory and 
Implications for Band-Gap Prediction, Phys. Rev. Lett. 100, 146401 (2008).

[128] J. D. Bernal and R. H. Fowler, A theory of water and ionic solution, with particular reference to hydrogen and hydroxyl ions, J. Chem. Phys. 1, 515 (1933).

[129] D. E. Hare and C. M. Sorensen, The density of supercooled water. II. Bulk samples cooled to the homogeneous nucleation limit, J. Chem. Phys. 87, 4840 (1987).

[130] O. A. Vydrov and T. Van Voorhis, Nonlocal van der Waals density functional: The simpler the better, J. Chem. Phys. 133, 244103 (2010).

[131] R. Sabatini, T. Gorni, and S. de Gironcoli, Nonlocal van der Waals density functional made simple and efficient, Phys. Rev. B 87, 041108(R) (2013).

[132] G. Miceli, S. de Gironcoli, and A. Pasquarello, Isobaric firstprinciples molecular dynamics of liquid water with nonlocal van der Waals interactions, J. Chem. Phys. 142, 034501 (2015).

[133] D. R. Hamann, $\mathrm{H}_{2} \mathrm{O}$ hydrogen bonding in density-functional theory, Phys. Rev. B 55, R10157 (1997).

[134] E. A. Engel, B. Monserrat, and R. J. Needs, Anharmonic Nuclear Motion and the Relative Stability of Hexagonal and Cubic Ice, Phys. Rev. X 5, 021033 (2015).

[135] T. K. Hirsch and L. Ojamäe, Quantum-chemical and forcefield investigations of ice Ih: Computation of proton-ordered structures and prediction of their lattice energies, J. Phys. Chem. B 108, 15856 (2004).

[136] Y. Tajima, T. Matsuo, and H. Suga, Phase transition in KOHdoped hexagonal ice, Nature (London) 299, 810 (1982).

[137] S. M. Jackson, V. M. Nield, R. W. Whitworth, M. Oguro, and C. C. Wilson, Single-crystal neutron diffraction studies of the structure of ice XI, J. Phys. Chem. B 101, 6142 (1997).

[138] D. R. Hamann, Optimized norm-conserving Vanderbilt pseudopotentials, Phys. Rev. B 88, 085117 (2013).

[139] M. J. van Setten, M. Giantomassi, E. Bousquet, M. J. Verstraete, D. R. Hamann, X. Gonze, and G.-M. Rignanese, The PSEUdoDoJo: Training and grading a 85 element optimized norm-conserving pseudopotential table, Comput. Phys. Commun. 226, 39 (2018).

[140] Z. H. Levine and D. C. Allan, Linear Optical Response in Silicon and Germanium including Self-Energy Effects, Phys. Rev. Lett. 63, 1719 (1989).

[141] S. Lebègue, B. Arnaud, M. Alouani, and P. E. Bloechl, Implementation of an all-electron GW approximation based on the projector augmented wave method without plasmon pole approximation: Application to $\mathrm{Si}, \mathrm{SiC}, \mathrm{AlAs}, \mathrm{InAs}, \mathrm{NaH}$, and KH, Phys. Rev. B 67, 155208 (2003).

[142] S. L. Adler, Quantum theory of the dielectric constant in real solids, Phys. Rev. 126, 413 (1962).
[143] N. Wiser, Dielectric constant with local field effects included, Phys. Rev. 129, 62 (1963).

[144] X. Gonze, B. Amadon, P.-M. Anglade, J.-M. Beuken, F. Bottin, P. Boulanger, F. Bruneval, D. Caliste, R. Caracas, and M. Côté, ABINIT: First-principles approach to material and nanosystem properties, Comput. Phys. Commun. 180, 2582 (2009).

[145] C. Friedrich, M. C. Müller, and S. Blügel, Band convergence and linearization error correction of all-electron $G W$ calculations: The extreme case of zinc oxide, Phys. Rev. B 83, 081101(R) (2011).

[146] P. Umari and A. Pasquarello, Polarizability and dielectric constant in density-functional supercell calculations with discrete $k$-point samplings, Phys. Rev. B 68, 085114 (2003).

[147] S. Fujita, S. Mae, and T. Matsuoka, Dielectric anisotropy in ice Ih at $9.7 \mathrm{GHz}$, Ann. Glaciol. 17, 276 (1993).

[148] T. Matsuoka, S. Fujita, S. Morishima, and S. Mae, Precise measurement of dielectric anisotropy in ice Ih at $39 \mathrm{GHz}$, J. Appl. Phys. 81, 2344 (1997).

[149] W. Chen and A. Pasquarello, First-principles determination of defect energy levels through hybrid density functionals and GW, J. Phys.: Condens. Matter 27, 133202 (2015).

[150] S. Falletta, J. Wiktor, and A. Pasquarello, Finite-size corrections of defect energy levels involving ionic polarization, Phys. Rev. B 102, 041115(R) (2020).

[151] P. Giannozzi, S. Baroni, N. Bonini, M. Calandra, R. Car, C. Cavazzoni, D. Ceresoli, G. L. Chiarotti, M. Cococcioni, and I. Dabo, QUANTUM ESPRESSO: A modular and opensource software project for quantum simulations of materials, J. Phys.: Condens. Matter 21, 395502 (2009).

[152] G. P. Johari, S. J. Jones, and F. C. Frank, Dielectric properties of polycrystalline $\mathrm{D}_{2} \mathrm{O}$ ice Ih (hexagonal), Proc. R. Soc. A 349, 467 (1976).

[153] N. Bjerrum, Structure and properties of ice, Science 115, 385 (1952).

[154] M. de Koning and A. Antonelli, On the trapping of Bjerrum defects in ice Ih: The case of the molecular vacancy, J. Phys. Chem. B 111, 12537 (2007).

[155] I. Popov, A. Puzenko, A. Khamzin, and Y. Feldman, The dynamic crossover in dielectric relaxation behavior of ice Ih, Phys. Chem. Chem. Phys. 17, 1489 (2015).

[156] T. J. Smart, T. A. Pham, Y. Ping, and T. Ogitsu, Optical absorption induced by small polaron formation in transition metal oxides: The case of $\mathrm{Co}_{3} \mathrm{O}_{4}$, Phys. Rev. Mater. 3, 102401(R) (2019).

[157] L. Kronik and S. Kümmel, Piecewise linearity, freedom from self-interaction, and a Coulomb asymptotic potential: Three related yet inequivalent properties of the exact density functional, Phys. Chem. Chem. Phys. 22, 16467 (2020). 